July 2008

\title{
Primordial Curvature Fluctuation and Its Non-Gaussianity in Models with Modulated Reheating
}

\author{
Kazuhide Ichikawa $^{1}$, Teruaki Suyama ${ }^{2}$, Tomo Takahashi ${ }^{3}$ \\ and Masahide Yamaguchi ${ }^{4,5}$ \\ ${ }^{1}$ Department of Physics and Astronomy, \\ University College London, London WC1E 6BT, UK \\ ${ }^{2}$ Institute for Cosmic Ray Research, University of Tokyo, Kashiwa 277-8582, Japan \\ ${ }^{3}$ Department of Physics, Saga University, Saga 840-8502, Japan \\ ${ }^{4}$ Department of Physics and Mathematics, \\ Aoyama Gakuin University, Sagamihara 229-8558, Japan \\ ${ }^{5}$ Department of Physics, Stanford University, Stanford CA 94305
}

\begin{abstract}
We investigate non-Gaussianity in the modulated reheating scenario where fluctuations of the decay rate of the inflaton generate adiabatic perturbations, paying particular attention to the non-linearity parameters $f_{\mathrm{NL}}, \tau_{\mathrm{NL}}$ and $g_{\mathrm{NL}}$ as well as the scalar spectral index and tensor-to-scalar ratio which characterize the nature of the primordial power spectrum. We also take into account the pre-existing adiabatic perturbations produced from the inflaton fluctuations. It has been known that the non-linearity between the curvature perturbations and the fluctuations of the decay rate can yield non-Gaussianity at the level of $f_{\mathrm{NL}} \sim \mathcal{O}(1)$, but we find that the non-linearity between the decay rate and the modulus field which determines the decay rate can generate much greater non-Gaussianity. We also discuss a consistency relation among non-linearity parameters which holds in the scenario and find that the modulated reheating yields a different one from that of the curvaton model. In particular, they both can yield a large positive $f_{\mathrm{NL}}$ but with a different sign of $g_{\mathrm{NL}}$. This provides a possibility to discriminate these two competitive models by looking at the sign of $g_{\mathrm{NL}}$. Furthermore, we work on some concrete inflation models and investigate in what cases models predict the spectral index and the tensor-to-scalar ratio allowed by the current data while generating large non-Gaussianity, which may have many implications for model-buildings of the inflationary universe.
\end{abstract}




\section{Introduction}

Inflation is a promising candidate to generate primordial density fluctuations as well as to solve the horizon and the flatness problems. Although many observational supports for the inflationary scenario have been accumulated, it is still unclear what mechanism is really responsible for producing primordial density fluctuations. We usually assume they are generated by fluctuations of the inflaton, but those of other scalar fields can produce the primordial fluctuations too. Such scalar fields generically exist in the extensions of the standard model of particle physics, which motivates the curvaton scenario [1-3] and modulated reheating scenarios $[4,5]$. One of interesting features of these scenarios is that primordial non-Gaussianity can possibly be very large, in contrast to the case of single field inflation models where only the inflaton is responsible for density fluctuations so that almost perfect Gaussian fluctuations arise. Therefore, non-Gaussianity can be a very powerful tool to identify the real source of the primordial fluctuations. In fact, there has been reported that non-Gaussianity is detected in the cosmic microwave background almost at $3 \sigma$ level [6] although this is not confirmed by the latest WMAP 5-year results [7]. (See also Refs. [8-10].) Further observations of WMAP and future observations such as Planck can give us more information on non-Gaussianity and serve to discriminate different scenarios.

In most works done thus far on this topic, only one source of the fluctuations has been considered. In general, however, since there exist a lot of scalar fields in a supergravity or superstring theory, different kinds of sources could contribute to the primordial fluctuations simultaneously. Therefore, it is interesting to consider models with mixed fluctuations from the inflaton and other sources. In such mixed scenarios, not only non-Gaussianity but also other features of the primordial fluctuations such as the amplitude, spectral index and tensor modes are affected in comparison to the case where the inflaton alone is the seed of fluctuations. Current cosmological data are already very precise regarding the scalar spectral index and the tensor-to-scalar ratio and they can severely constrain models of inflation. Some models of inflation are considered to have already been excluded [7]. For example, chaotic inflation with the higher order polynomial potential is excluded at more than $95 \%$ confidence level. But, such constraints may be evaded by adding another source of fluctuations. In fact, it is shown that some models of inflation which are disfavored by the data can be liberated by adding the curvaton contribution [11-18]. In particular, in Ref. [17], it has been studied in what cases models of inflation can be relaxed by adding fluctuations from the curvaton in some detail assuming some concrete inflation models focusing on the spectral index and the tensor-to-scalar ratio. Furthermore, in Ref. [19], it was also discussed in what cases/models large non-Gaussianity can be generated satisfying the constraints on the scale dependence and tensor modes of primordial fluctuations in the mixed scenario.

The modulated reheating scenario has been paid much attention recently as interesting other source of fluctuations and large non-Gaussianity but it is not so rigorously investigated as the curvaton mechanism in particular as regards mixed scenarios with the 
inflaton. Therefore, in this paper, we consider the mixed models where fluctuations from the inflaton and the modulated coupling can both contribute to the present cosmic density fluctuations. Then, we study the effects of the contribution of the modulated coupling on inflationary parameters such as the spectral index and the tensor-to-scalar ratio and compare with the WMAP 5-year results. Furthermore, we also discuss in what cases large non-Gaussianity can be generated satisfying the observational constraints on the scale dependence and tensor modes of primordial fluctuations in such a mixed scenario.

The structure of this paper is as follows. In the next section, we will give the expressions of the decay rate of the inflaton into radiation for various types of interactions between the inflaton and other fields and for various inflaton potentials, which will be used in the subsequent sections. In section 3, we will provide the $e$-folding number from the time when the current cosmological scales crossed the Hubble horizon during inflation to the time after the inflaton decays, which enables us to evaluate the curvature perturbations generated in this scenario. Then we give the expressions for the scalar spectral index, tensor-to-scalar ratio and three non-linearity parameters in section 4. With the formalism summarized in section 4, we work on some specific inflation models to compare the predictions of these inflationary parameters with observations, paying particular attention to in what cases non-Gaussianity can be very large. For inflation models which are considered to have already been excluded by the data, we also give discussions in what case the contribution from the modulated reheating can liberate the model. The final section is devoted to the conclusion and summary of this paper.

\section{Decay rate of the inflaton}

After inflation, the inflaton oscillates around the minimum of its potential and the period of the oscillations is much shorter than the expansion time characterized by the Hubble parameter. The energy density of the universe at that time is stored in the form of oscillation energy of the inflaton. Since the universe should become radiation dominated before the time of big-bang nucleosynthesis, which is required to be consistent with observations, the inflaton must decay into radiation at some time after inflation. Regarding interactions between inflaton and radiation, we consider the following Lagrangian,

$$
\mathcal{L}_{\text {int }} \supset-\sum_{a} y_{a}(\sigma) \phi \bar{\psi}_{a} \psi_{a}-\sum_{a} M_{a}(\sigma) \phi \chi_{a}^{2}-\sum_{a} h_{a}(\sigma) \phi^{2} \chi_{a}^{2}
$$

where $\phi$ is the inflaton and $\chi_{a}$ and $\psi_{a}$ are scalar and spinor fields which constitute radiation ( $a$ represents the species of the particles). In the modulated reheating scenario, the coupling constants $y_{a}, M_{a}$ and $h_{a}$ are functions of a scalar field which we denote as $\sigma$. Although the number of such scalar fields is not necessarily one, we consider only one modulus to avoid inessential complexity.

The oscillations of the inflaton act as a periodically changing external field on $\chi_{a}$ and $\psi_{a}$ fields. This external field creates $\chi_{a}$ or $\psi_{a}$ particles out of the vacuum. Due to the 
total energy density conservation, the energy of created particles are compensated by the loss of the oscillation energy of the inflaton. Hence this process can be regarded as the particle production from the inflaton decay.

Let us suppose that the inflaton potential around the minimum can be well approximated by a polynomial form as $V(\phi) \propto \phi^{2 n}$ with $n$ being a positive integer. Denoting the inflaton energy density as $\rho_{\phi}$, the decay rate of the inflaton to the lowest order in the coupling constants is given by

$$
\Gamma_{\phi}^{(n)}(\sigma)=\sum_{a} A_{n} \frac{y_{a}^{2}(\sigma)}{8 \pi} m_{\phi}^{\mathrm{eff}}+\sum_{a} B_{n} \frac{M_{a}^{2}(\sigma)}{8 \pi m_{\phi}^{\mathrm{eff}}}+\sum_{a} C_{n} \frac{h_{a}^{2}(\sigma)}{8 \pi\left(m_{\phi}^{\mathrm{eff}}\right)^{3}} \rho_{\phi},
$$

where $m_{\phi}^{\text {eff }}$ is the effective mass of the inflaton defined by

$$
\left(m_{\phi}^{\mathrm{eff}}\right)^{2}=\left.V_{\phi \phi}\right|_{\phi=\bar{\phi}}
$$

with $\bar{\phi}$ being the amplitude of the oscillations. Here and hereafter, the subscript $\phi$ of the potential $V$ represents the derivative with respect to $\phi . A_{n}, B_{n}$ and $C_{n}$ are numerical coefficients of $\mathcal{O}(1 \sim 100)$. The explicit values of these are given in the appendix $\mathrm{A}$.

\section{The number of $e$-folding}

In the following, the $e$-folding number especially plays an important role in two aspects. First of all, when we calculate observables such as the amplitude, spectral index, nonGaussianity and so on of the curvature perturbations, we have to know when the cosmological scales exited the Hubble horizon during inflation, i.e. the $e$-folding number from the time of horizon crossing during inflation to the present. To this end, we need to know how the universe evolved from the inflationary universe into the standard radiation dominated universe, which obviously requires our knowledge of how the universe is reheated by the inflaton decay. Another aspect is that knowledge of the $e$-folding number enables us to calculate the curvature perturbations to any order in the perturbative expansion without invoking complicated perturbed equations but using the so-called $\delta N$ formalism [20-23]. All the information regarding various observables of the curvature perturbations is contained in the $e$-folding number. In this section, we give an expression for the $e$-folding

number from the time $t_{*}$ when the cosmological scale crossed the horizon during inflation to the time $t_{f}$ when the universe is completely reheated by the inflaton decay. After $t_{f}$, we assume that the universe evolves according to the standard hot Big-Bang model.

Let us denote the $e$-folding number from $t_{*}$ to $t_{f}$ as $N\left(t_{f}, t_{*}, \phi_{*}, \sigma_{*}\right)$. Here $\phi_{*}$ and $\sigma_{*}$ are values of $\phi$ and $\sigma$ at $t=t_{*}$. For later convenience, we divide $N\left(t_{f}, t_{*}, \phi_{*}, \sigma_{*}\right)$ into two parts as

$$
N\left(t_{f}, t_{*}, \phi_{*}, \sigma_{*}\right)=N\left(t_{\mathrm{end}}, t_{*}, \phi_{*}\right)+N\left(t_{f}, t_{\mathrm{end}}, \sigma_{*}\right),
$$


where $t_{\text {end }}$ is the time at the end of inflation. Hence, on the right-hand side, the first term represents the $e$-folding number during inflation and the second term remaining one after the inflation. In writing this equation, we have implicitly assumed that the mass and vacuum expectation value of the modulus are sufficiently small and the background dynamics during inflation is completely determined by the inflaton alone, which means that the first term on the right-hand side is a function of $\phi_{*}$ only. Meanwhile, the second term depends only on $\sigma_{*}$.

By using the slow roll approximation, we write the first term as

$$
N\left(t_{\mathrm{end}}, t_{*}, \phi_{*}\right)=\int_{t_{*}}^{t_{\mathrm{end}}} H d t \simeq-\frac{1}{M_{\mathrm{pl}}^{2}} \int_{\phi_{*}}^{\phi_{\mathrm{end}}} \frac{V}{V_{\phi}} d \phi,
$$

where $\phi_{*}$ and $\phi_{\text {end }}$ represent the scalar field values at corresponding epochs. To give a more concrete expression for $N\left(t_{\text {end }}, t_{*}, \phi_{*}\right)$, we need to specify the potential for the inflaton.

Let us next consider the second term $N\left(t_{f}, t_{\text {end }}, \sigma_{*}\right)$. When the slow-roll conditions are violated, the inflaton starts to oscillate around its minimum. After several oscillations, the energy density of the inflaton can be well approximated by that of a perfect fluid with a constant equation of state. If the potential around the minimum is written as $V \propto \phi^{2 n}$, its energy density decreases as $\rho_{\phi} \propto a^{-6 n /(n+1)}$. For the discussion of the curvature perturbations in the next section, we further decompose the second term into two parts. Taking $t_{c}$ as a time after several oscillations of the inflaton but well before the time of decay, $N\left(t_{f}, t_{\text {end }}, \sigma_{*}\right)$ can be divided as

$$
N\left(t_{f}, t_{\mathrm{end}}, \sigma_{*}\right)=N\left(t_{c}, t_{\mathrm{end}}\right)+N\left(t_{f}, t_{c}, \sigma_{*}\right) .
$$

Note that the first term on the right-hand side does not depend on $\sigma_{*}$ as long as $t_{c}$ is taken to be sufficiently before the time of the decay. As will become clear later, $N\left(t_{c}, t_{\text {end }}\right)$ is irrelevant to the curvature perturbations. However, we have to take into account it when we calculate the epoch when the reference scale at present, which is taken to be $k=0.002 \mathrm{Mpc}^{-1}$ for our analysis, exited the horizon during inflation. In general, we need a numerical calculation to evaluate $N\left(t_{c}, t_{\text {end }}\right)$ and do not discuss this term further here.

Regarding $N\left(t_{f}, t_{c}, \sigma_{*}\right)$, for our discussion in the following, we write it as

$$
N\left(t_{f}, t_{c}, \sigma_{*}\right)=\frac{1}{4} \log \frac{\rho_{c}}{\rho_{f}}+Q\left[\Gamma_{\phi}\left(\sigma_{*}, t_{c}\right) / H_{c}\right]
$$

where $\rho_{c}$ and $\rho_{f}$ are total energy densities of the universe at $t_{c}$ and $t_{f}$ respectively. This equation should be understood as a definition of the function $Q$. Note that $Q$ depends on the variable $x \equiv \Gamma\left(\sigma_{*}, t_{c}\right) / H_{c}$ alone, which can be confirmed by a dimensional analysis of the background evolution equations. Since the dependence of $Q$ on $x$ differs depending on the expansion law of the background space-time, i.e. the power $2 n$ that determines the form of the inflaton potential, and also on the dominant decay channel of the inflaton, we need to follow the background evolution for each case. In principle, we have to evaluate it by numerical calculations. However, we can make approximate analytic estimates for $Q(x)$ 


\begin{tabular}{|c|c|c|c|}
\hline $\mathcal{L}_{\text {int }}$ & $n=1$ & $n=2$ & $n=3$ \\
\hline$-y \phi \psi \psi$ & $-\frac{1}{6}$ & 0 & $\frac{1}{6}$ \\
\hline$-M \phi \chi \chi$ & $-\frac{1}{6}$ & 0 & $\frac{1}{30}$ \\
\hline$-h \phi^{2} \chi^{2}$ & - & 0 & $\frac{1}{18}$ \\
\hline
\end{tabular}

Table 1: We list the analytic values of $a_{0}$ defined by $Q(x)=a_{0} \log x$ for various inflaton potential $V(\phi) \propto \phi^{2 n}$ and dominant interactions. Notice that these values are in good agreement with numerically obtained ones, in particular for small values of $x$. If $n=1$, the inflaton cannot decay only with the four-point interaction $-h \phi^{2} \chi^{2}$.

when $x \ll 1$. We found that $Q(x)$ can be well approximated with the form $Q(x)=a_{0} \log x$ where $a_{0}$ is a numerical constant which depends on the interaction between the inflaton and matter and the potential for the inflaton. In Table 1, the values of $a_{0}$ are listed for three different powers for the inflaton potential and three different interactions, which are obtained by analytic estimates where the sudden-decay approximation is adopted. The derivation of these analytic values is given in Appendix B. We have also calculated them numerically and found that the differences between analytic estimate and numerical one are at most $10 \%$ for $x<10^{-6}$, which can be seen from Fig. 11 where the relative error for the case with the inflation potential being $V \propto \phi^{6}$ and three types of interaction listed in Table 1.

In the following analysis, we consider the cases with $n=1,2$ and 3 . Here we briefly discuss the tendency of $Q$ for these cases in order. When $n=1$, the inflaton potential can be written as $V(\phi)=\frac{m^{2}}{2} \phi^{2}$. In this case, we have $m_{\phi}^{\text {eff }}=m$ and the decay rate becomes independent of time for the interactions such as $-y \phi \bar{\psi} \psi$ and $-M \phi \chi^{2}$. Thus we obtain the same value of $Q$ for these interactions. Meanwhile, if the dominant decay occurs through the four-point interaction, the inflaton cannot decay completely into radiation simply because the decay rate which is proportional to the Hubble parameter squared decreases faster than the Hubble parameter. Thus in this case, the universe cannot be reheated. Hence we do not consider a four-point interaction case for the quadratic potential. When $n=2, \rho_{\phi}$ behaves the same as the energy density of radiation. Hence the universe expands in exactly the same way as the background space-time even if the decay rate of the inflaton slightly deviates from the background value\#. This means that $Q=0$ for any interactions. For the case with $n=3$, since the effective mass $m_{\phi}^{\text {eff }}$ changes in time, the decay rate evolves differently for different types of a dominant interaction, which gives a different form of $Q$.

Also note that since the coefficient of $\log x$ in $Q(x)$ is negative (positive) for $n=1$ ( $n=$ 3 ), the function $Q(x)$ itself becomes positive (negative) for $n=1(n=3)$ when $x \leq 1$. From the definition of $Q$ (see Eq. (7)), $Q$ represents the deviation of the $e$-folding number

\#1 This is not true if preheating occurs. In Ref. [24], it was shown that the universe can evolve differently at different locations separated by the super-horizon distance during preheating because of the persisting isocurvature perturbations at the end of inflation. 


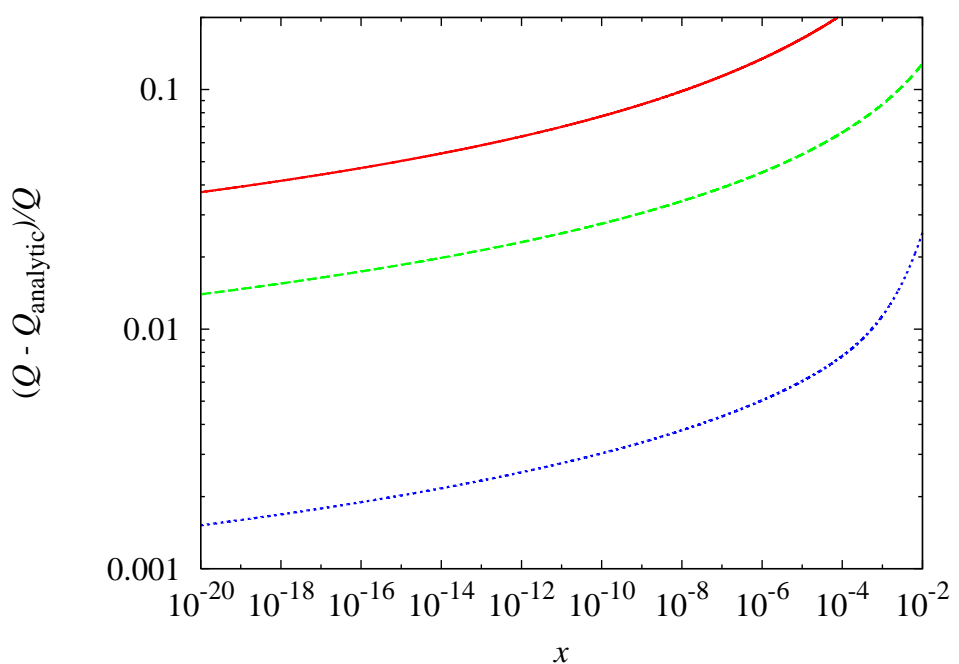

Figure 1: The relative error for the function $Q(x)$ between the one obtained by analytic and numerical methods for the cases with the interaction $-y \phi \bar{\psi} \psi$ (red solid line), $-M \phi \chi^{2}$ (green dashed line) and $-h \phi^{2} \chi^{2}$ (blue dotted line). Here we assumed $V \propto \phi^{6}$ for the inflaton potential. For the cases with the quadratic potential $V \propto \phi^{2}$, the errors are smaller than those given in this figure.

from the radiation dominated universe. Because energy density of dust decays more slowly than the radiation energy density, the more dust gives the more $e$-folding number until the total energy density decreases to a fixed value, which means the positive $Q$. Meanwhile, because the energy density of the inflaton oscillating in the sextic potential decays as $a^{-9 / 2}$, it decreases more rapidly than the energy density of radiation. Hence $Q$ becomes negative for the sextic potential.

In closing this section, we write down the expression for $N\left(t_{f}, t_{*}, \phi_{*}, \sigma_{*}\right)$ :

$$
N\left(t_{f}, t_{*}, \phi_{*}, \sigma_{*}\right)=-\frac{1}{M_{\mathrm{pl}}^{2}} \int_{\phi_{*}}^{\phi_{\mathrm{end}}} d \phi \frac{V}{V_{\phi}}+\frac{1}{4} \log \frac{\rho_{c}}{\rho_{f}}+N\left(t_{c}, t_{\mathrm{end}}\right)+Q\left[\Gamma_{\phi}\left(\sigma_{*}\right) / H_{0}\right],
$$

which forms the basis in calculating various observables in the subsequent sections.

\section{Observables}

\subsection{Curvature perturbation}

To calculate the curvature perturbation, we make use of the $\delta N$ formalism [20-23]. In this formalism, the primordial curvature perturbation $\zeta$ on the uniform energy density hypersurface at the time $t=t_{f}$ is given by differentiating the $e$-folding number $N\left(t_{f}, t_{*}, \phi_{*}, \sigma_{*}\right)$ 
in Eq. (8) with respect to $\phi_{*}$ and $\sigma_{*}$,

$$
\begin{array}{r}
\zeta \approx \frac{1}{M_{\mathrm{pl}}^{2}} \frac{V}{V_{\phi}} \delta \phi_{*}+\frac{1}{2 M_{\mathrm{pl}}^{2}}\left(1-\frac{V V_{\phi \phi}}{V_{\phi}^{2}}\right) \delta \phi_{*}^{2}+\frac{1}{6 M_{\mathrm{pl}}^{2}}\left(-\frac{V_{\phi \phi}}{V_{\phi}}-\frac{V V_{\phi \phi \phi}}{V_{\phi}^{2}}+2 \frac{V V_{\phi \phi}^{2}}{V_{\phi}^{3}}\right) \delta \phi_{*}^{3} \\
+Q_{\sigma} \delta \sigma_{*}+\frac{1}{2} Q_{\sigma \sigma} \delta \sigma_{*}^{2}+\frac{1}{6} Q_{\sigma \sigma \sigma} \delta \sigma_{*}^{3},
\end{array}
$$

where $\delta \phi_{*}$ and $\delta \sigma_{*}$ are the perturbations of $\phi_{*}$ and $\sigma_{*}$ on the flat slicing. For the purpose of this paper, we include the terms up to cubic order in the perturbations of scalar fields. If we choose $t_{f}$ well after the reheating, then $\zeta\left(t_{f}\right)$ gives primordial adiabatic perturbations.

Using $x=\Gamma_{\phi}\left(\sigma_{*}, t_{c}\right) / H_{c}$, the derivatives of $Q$ with respect to $\sigma$ can be expressed using $\Gamma_{\phi}$ as

$$
\begin{aligned}
Q_{\sigma}= & x Q^{\prime}(x) \frac{\Gamma_{\sigma}}{\Gamma}=A(x) \frac{\Gamma_{\sigma}}{\Gamma}, \\
Q_{\sigma \sigma} & =x Q^{\prime}(x) \frac{\Gamma_{\sigma \sigma}}{\Gamma}+x^{2} Q^{\prime \prime}(x) \frac{\Gamma_{\sigma}^{2}}{\Gamma^{2}}=A(x) \frac{\Gamma_{\sigma \sigma}}{\Gamma}+B(x) \frac{\Gamma_{\sigma}^{2}}{\Gamma^{2}}, \\
Q_{\sigma \sigma \sigma} & =x Q^{\prime}(x) \frac{\Gamma_{\sigma \sigma \sigma}}{\Gamma}+3 x^{2} Q^{\prime \prime}(x) \frac{\Gamma_{\sigma} \Gamma_{\sigma \sigma}}{\Gamma^{2}}+x^{3} Q^{\prime \prime \prime}(x) \frac{\Gamma_{\sigma}^{3}}{\Gamma^{3}} \\
& =A(x) \frac{\Gamma_{\sigma \sigma \sigma}}{\Gamma}+3 B(x) \frac{\Gamma_{\sigma} \Gamma_{\sigma \sigma}}{\Gamma^{2}}+C(x) \frac{\Gamma_{\sigma}^{3}}{\Gamma^{3}} .
\end{aligned}
$$

Here we defined the function as $A(x) \equiv x Q(x)^{\prime}, B(x)=x^{2} Q(x)^{\prime \prime}$ and $C(x)=x^{3} Q(x)^{\prime \prime \prime}$ and denote the derivatives of $\Gamma_{\phi}$ with respect to $\sigma_{*}$ as $\Gamma_{\sigma}, \Gamma_{\sigma \sigma}$, and $\Gamma_{\sigma \sigma \sigma}$. When $x \ll 1$, which we consider in the followings, these functions become almost independent of $x$, namely $Q$ can be well approximated by $Q(x)=a_{0} \log x$ (see Table 1). In this case, the functions defined above become constant and can be written as $A=a_{0}, B=-a_{0}$ and $C=2 a_{0}$.

\subsection{Power spectrum}

The power spectrum $P_{\zeta}$ of the curvature perturbations is defined by

$$
\left\langle\zeta_{\vec{k}_{1}} \zeta_{\vec{k}_{2}}\right\rangle_{c}=(2 \pi)^{3} P_{\zeta}\left(k_{1}\right) \delta\left(\vec{k}_{1}+\vec{k}_{2}\right)
$$

where $\langle\cdots\rangle_{c}$ means that we take connected parts of $\langle\cdots\rangle$.

By using Eq. (9), we can express $\mathcal{P}_{\zeta}(k) \equiv k^{3} P_{\zeta}(k) / 2 \pi^{2}$ to the leading order in $\delta \phi_{*}, \delta \sigma_{*}$ as

$$
\mathcal{P}_{\zeta}(k)=\frac{1}{2 \epsilon}\left(\frac{H_{*}}{2 \pi M_{\mathrm{pl}}}\right)^{2}(1+R),
$$

where $\epsilon$ is a slow-roll parameter defined by,

$$
\epsilon \equiv \frac{M_{\mathrm{pl}}^{2}}{2} \frac{V_{\phi}^{2}}{V^{2}}
$$


Furthermore $R \equiv 2 \epsilon A(x)^{2} M_{\mathrm{pl}}^{2} \Gamma_{\sigma}^{2} / \Gamma^{2}$ is the square of the ratio of the curvature perturbation from modulated reheating to that from the inflaton, i.e., $\zeta_{\text {mod }}^{2} / \zeta_{\text {inf }}^{2}$. Thus the limit $R \rightarrow 0$ $(R \rightarrow \infty)$ corresponds to the case where the curvature perturbation is sourced only by the inflaton (modulus) fluctuations. For discussion in the following, here we give the definition of another slow-roll parameter $\eta$ :

$$
\eta \equiv M_{\mathrm{pl}}^{2} \frac{V_{\phi \phi}}{V}
$$

\subsection{Bispectrum}

The bispectrum $B_{\zeta}$ is defined by

$$
\left\langle\zeta_{\vec{k}_{1}} \zeta_{\vec{k}_{2}} \zeta_{\vec{k}_{3}}\right\rangle_{c}=(2 \pi)^{3} B_{\zeta}\left(k_{1}, k_{2}, k_{3}\right) \delta\left(\vec{k}_{1}+\vec{k}_{2}+\vec{k}_{3}\right) .
$$

During inflation, both $\phi$ and $\sigma$ are slowly-rolling. It is known that the non-Gaussianity of $\zeta$ coming from the intrinsic non-Gaussianities of $\delta \phi_{*}$ and $\delta \sigma_{*}$ is far below the observational sensitivity. Hence we can treat $\delta \phi_{*}$ and $\delta \sigma_{*}$ as uncorrelated Gaussian random fields with the same amplitude.

If we parameterize $B_{\zeta}$ by the dimensionless parameter $f_{\mathrm{NL}}$ by

$$
B_{\zeta}\left(k_{1}, k_{2}, k_{3}\right)=\frac{6}{5} f_{\mathrm{NL}}\left(P_{\zeta}\left(k_{1}\right) P_{\zeta}\left(k_{2}\right)+P_{\zeta}\left(k_{2}\right) P_{\zeta}\left(k_{3}\right)+P_{\zeta}\left(k_{3}\right) P_{\zeta}\left(k_{1}\right)\right),
$$

then $f_{\mathrm{NL}}$ can be written as

$$
\frac{6}{5} f_{\mathrm{NL}}=\frac{R^{2}}{A(x)(1+R)^{2}}\left(\frac{B(x)}{A(x)}+\frac{\Gamma \Gamma_{\sigma \sigma}}{\Gamma_{\sigma}^{2}}\right)+\mathcal{O}(\epsilon, \eta) .
$$

The first term $A(x)^{-2} B(x) R^{2} /(1+R)^{2}$ which is independent of how the decay rate $\Gamma$ depends on $\sigma$ represents the non-Gaussianity coming from the non-linearity between $\zeta$ and $\delta \Gamma$. Because the function $R^{2} /(1+R)^{2}$ is suppressed by $R^{2}$ for $R \ll 1$ and approaches 1 for $R \gg 1$, the magnitude of the first term is at most $\left|A^{-2} B\right|=\mathcal{O}(1 \sim 10)$. For example, if the potential is given by a quadratic term, we have $\left|A^{-2} B\right| \simeq 6$ (see Table 11), which yields $f_{\mathrm{NL}}=5$. The non-linearity between $\zeta$ and $\delta \Gamma$ gives the positive (negative) $f_{\mathrm{NL}}$ for quadratic (sextic) inflaton potential.

On the other hand, the second term which represents the non-Gaussianity coming from the non-linearity between $\Gamma$ and $\sigma$ depends on the detailed form of $\Gamma(\sigma)$. We see that very large non-Gaussianity $\left|f_{\mathrm{NL}}\right| \gg 1$ can be generated only when $\left|\Gamma \Gamma_{\sigma \sigma} / \Gamma_{\sigma}^{2}\right| \gg 1$ is satisfied.

\subsection{Trispectrum}

The trispectrum $T_{\zeta}$ is defined by

$$
\left\langle\zeta_{\vec{k}_{1}} \zeta_{\vec{k}_{2}} \zeta_{\vec{k}_{3}} \zeta_{\vec{k}_{4}}\right\rangle_{c}=(2 \pi)^{3} T_{\zeta}\left(k_{1}, k_{2}, k_{3}, k_{4}\right) \delta\left(\vec{k}_{1}+\vec{k}_{2}+\vec{k}_{3}+\vec{k}_{4}\right) .
$$




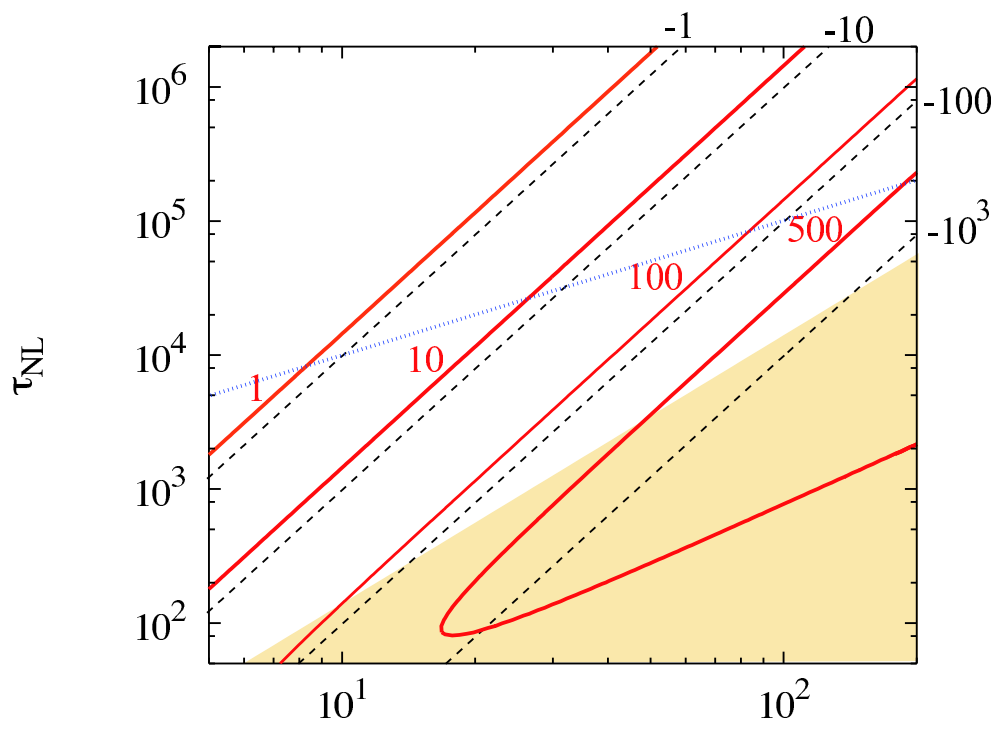

$\mathrm{f}_{\mathrm{NL}}$

Figure 2: Consistency relations among three non-linearity parameters are shown for the case with modulated reheating corresponding to Eq. (25) (red solid line) and the curvaton model for the case where the curvaton decays before it dominates the universe corresponding to Eq. (77) in Ref [19] (black dashed line). In this figure, the consistency relation is presented as contours of $g_{\mathrm{NL}}$ in the $f_{\mathrm{NL}}-\tau_{\mathrm{NL}}$ plane. The line for models with "ungaussiton" [26] is also shown (blue dotted line). For this model, the relation between $f_{\mathrm{NL}}$ and $\tau_{\mathrm{NL}}$ is given, thus it is irrelevant to the value of $g_{\mathrm{NL}}$. Notice that the inequality Eq. (24) should hold for the above mentioned scenarios. Thus we also show a region violating this inequality with shade.

If we parameterize $T_{\zeta}$ by the two dimensionless parameters $\tau_{\mathrm{NL}}$ and $g_{\mathrm{NL}}$ as

$$
\begin{aligned}
T_{\zeta}\left(k_{1}, k_{2}, k_{3}, k_{4}\right)= & \tau_{\mathrm{NL}}\left(P_{\zeta}\left(k_{13}\right) P_{\zeta}\left(k_{3}\right) P_{\zeta}\left(k_{4}\right)+11 \text { perms. }\right) \\
& +\frac{54}{25} g_{\mathrm{NL}}\left(P_{\zeta}\left(k_{2}\right) P_{\zeta}\left(k_{3}\right) P_{\zeta}\left(k_{4}\right)+3 \text { perms. }\right),
\end{aligned}
$$

then these parameters are given by

$$
\begin{aligned}
& \tau_{\mathrm{NL}}=\frac{R^{3}}{A(x)^{2}(1+R)^{3}}\left(\left(\frac{B(x)}{A(x)}\right)^{2}-\frac{\Gamma \Gamma_{\sigma \sigma}}{\Gamma_{\sigma}^{2}}\right)^{2}+\mathcal{O}\left(\epsilon^{2}, \epsilon \eta, \eta^{2}\right) \\
& \quad=\frac{36(1+R)}{25 R} f_{\mathrm{NL}}^{2}+\mathcal{O}\left(\epsilon^{2}, \epsilon \eta, \eta^{2}\right), \\
& \frac{54}{25} g_{\mathrm{NL}}=\frac{R^{3}}{A(x)^{2}(1+R)^{3}}\left(\frac{C(x)}{A(x)}+\frac{3 B(x)}{A(x)} \frac{\Gamma \Gamma_{\sigma \sigma}}{\Gamma_{\sigma}^{2}}+\frac{\Gamma^{2} \Gamma_{\sigma \sigma \sigma}}{\Gamma_{\sigma}^{3}}\right)+\mathcal{O}\left(\epsilon \eta, \xi^{2}, \eta^{2}\right) .
\end{aligned}
$$


From Eqs. (19) and (22), neglecting the terms of the slow-roll order, we find that

$$
\tau_{\mathrm{NL}} \geq \frac{36}{25} f_{\mathrm{NL}}^{2}
$$

In Ref. [25], it is shown that this inequality holds in more general setting, i.e. for any cases where the leading non-Gaussianity comes from super-horizon evolution.

As for $g_{\mathrm{NL}}$, the non-linearity between $\zeta$ and $\delta \Gamma$ always gives positive values for $g_{\mathrm{NL}}$ irrespective of the inflaton potential and the dominant interaction. When the non-linearity between $\delta \Gamma$ and $\delta \sigma$ cannot be neglected, $g_{\mathrm{NL}}$ can be negative.

If the $\Gamma_{\sigma \sigma \sigma}$ term in $g_{\mathrm{NL}}$ can be neglected and $x \ll 1$, all the three non-linear parameters are functions of $R$ and $\Gamma \Gamma_{\sigma \sigma} / \Gamma_{\sigma}^{2}$. This means that we have a universal relation among the

non-linearity parameters independent of $R$ and $\Gamma \Gamma_{\sigma \sigma} / \Gamma_{\sigma}^{2}$. The explicit form of such a relation can be written as

$$
g_{\mathrm{NL}}=-\frac{864}{625 a_{0}^{2}} \frac{f_{\mathrm{NL}}^{6}}{\tau_{\mathrm{NL}}^{3}}-\frac{12}{5 a_{0}} \frac{f_{\mathrm{NL}}^{3}}{\tau_{\mathrm{NL}}}
$$

In particular, if the potential around the minimum is quadratic, then we have $a_{0}=-\frac{1}{6}$ and the relation above reduces to

$$
g_{\mathrm{NL}}=-\frac{31104}{625} \frac{f_{\mathrm{NL}}^{6}}{\tau_{\mathrm{NL}}^{3}}+\frac{72}{5} \frac{f_{\mathrm{NL}}^{3}}{\tau_{\mathrm{NL}}}
$$

By checking this consistency relation from observations, we can discriminate this scenario from other ones that also generate large non-Gaussianity. In Fig. 2, we show the contours of $g_{\mathrm{NL}}$ as a function of $f_{\mathrm{NL}}$ and $\tau_{\mathrm{NL}}$ given in Eq. (26). For comparison, we also show the relation among the non-linearity parameters which holds for other scenarios which can generate large non-Gaussianity such as models with mixed inflaton and curvaton for the case with a large positive $f_{\mathrm{NL}}$ [19] and "ungaussiton" [26]. Interestingly, while the curvaton gives negative $g_{\mathrm{NL}}$, the modulated reheating gives positive one (if the potential is quadratic) if $f_{\mathrm{NL}}$ is large positive. Hence just the determination of the sign of $g_{\mathrm{NL}}$ enables us to discriminate these two competitive models in this case. We also plot the relation between $f_{\mathrm{NL}}$ and $\tau_{\mathrm{NL}}$ in the "ungaussiton" model where the relation between $\tau_{\mathrm{NL}}$ and $f_{\mathrm{NL}}$ is given irrespective of $g_{\mathrm{NL}}$. Thus only one line is drawn for this model.

\subsection{The scalar spectral index and the tensor-to-scalar ratio}

In this subsection, we will give the expressions for the scalar spectral index and tensor-toscalar ratio. First of all, the scalar spectral index $n_{s}$ is given by

$$
\begin{aligned}
n_{s}-1 & \left.\equiv \frac{d \log \mathcal{P}_{\zeta}}{d \log k}\right|_{k=a H} \\
& =-2 \epsilon-\frac{4 \epsilon-2 \eta}{1+R} .
\end{aligned}
$$


During inflation, the tensor mode is also generated and its power spectrum can be written as

$$
\mathcal{P}_{T}=8\left(\frac{H}{2 \pi M_{\mathrm{pl}}}\right)^{2} .
$$

To characterize the size of the tensor mode, the tensor-to-scalar ratio is usually used, which is given by

$$
r \equiv \frac{\mathcal{P}_{T}}{\mathcal{P}_{\zeta}}=\frac{16 \epsilon}{1+R} .
$$

\section{Inflationary predictions and comparison with ob- servations}

In this section, we discuss the five inflationary parameters, the scalar spectral index, tensorto-scalar ratio and three non-linearity parameters $\left(n_{s}, r, f_{\mathrm{NL}}, \tau_{\mathrm{NL}}, g_{\mathrm{NL}}\right)$ in models where fluctuations from modulated coupling and inflaton can both contribute to the primordial curvature perturbations. With regard to $n_{s}$ and $r$, current cosmological observations give severe constraints on these quantities, thus we also compare the predictions for $n_{s}$ and $r$ with the WMAP 5-year data [7,27]. For the purpose of this paper, we consider the chaotic inflation models with some polynomials for definiteness. However, before we discuss its inflationary predictions, here we make some comments on the cases with other inflation models in a mixed scenario. If we take the new inflation models, its effects are expected to be very small since the new inflation models usually give a negligibly small value of the slow-roll parameter $\epsilon$. Since $\epsilon$ appears in $R=2 \epsilon A(x)^{2} M_{\mathrm{pl}}^{2} \Gamma_{\sigma}^{2} / \Gamma^{2}$ which represents the size of the contribution from fluctuations from modulated reheating, $R$ becomes very small when $\epsilon$ is negligibly small. This fact has already been pointed out in Ref. [19] for a mixed model of the inflaton and curvaton. Hence, it is unlikely that fluctuations from modulated reheating dominate the total curvature perturbations in the new inflation model, in which we recover the usual standard formula for the inflationary parameters and the effects of modulated reheating can be negligible. As another possible inflation model, we can also assume the hybrid inflation model. However, as is also discussed in Ref. [19], the case of hybrid inflation leads to the similar result as that of chaotic inflation. Thus we omit them in this paper. Regarding the interactions between the inflaton and matter, we adopt the Yukawa interactions, just to be concrete. We can straightforwardly analyze the case with other interactions by simply replacing the numerical factor $A(x), B(x)$ and $C(x)$ appearing in the expression with the corresponding values (see Table 1).

Even after we fix the form of the inflaton potential and the interactions for reheating, we have still a degree of freedom of how the coupling constant $y$ depends on the modulus 
$\sigma$. In the following, we assume that $y_{a}(\sigma)$ can be written as $\# 2$.

$$
y_{a}(\sigma)=y_{a}^{0}\left(1+\alpha_{a} \frac{\sigma}{M}+\beta_{a} \frac{\sigma^{2}}{M^{2}}+\cdots\right),
$$

where $\alpha_{a}$ and $\beta_{a}$ are $\mathcal{O}(1)$ coefficients. $M$ is some energy scale and we assume that $|\sigma| \ll M$. To be definite, we truncate the expansion of $y_{a}(\sigma)$ at the second order in $\sigma$. Then the decay rate can be also truncated at the quadratic order in $\sigma$,

$$
\Gamma=\Gamma_{0}\left(1+\alpha \frac{\sigma}{M}+\beta \frac{\sigma^{2}}{M^{2}}\right),
$$

where $\alpha$ and $\beta$ are also $\mathcal{O}(1)$ coefficients.

From Eq. (31), we have

$$
\frac{\Gamma \Gamma_{\sigma \sigma}}{\Gamma_{\sigma}^{2}} \simeq \frac{2 \beta}{\left(\alpha+\frac{2 \beta \sigma}{M}\right)^{2}}
$$

If $\alpha$ is $\mathcal{O}(1)$, then this equation further reduces to $\simeq 2 \beta / \alpha^{2}$. From Eq. (19), the nonlinearity between $\Gamma$ and $\sigma$ gives $f_{\mathrm{NL}} \simeq 2 \beta /\left(A(x) \alpha^{2}\right)$ for $R \gg 1$. Since $\left|A(x)^{-1}\right|$ is $\mathcal{O}(1 \sim$ $10),\left|f_{\mathrm{NL}}\right|=\mathcal{O}(10 \sim 100)$ can be achieved by setting $2 \beta / \alpha^{2}$ to be $\mathcal{O}(10)$, which is quite possible while satisfying $\alpha, \beta=\mathcal{O}(1)$. Notice that, to obtain a large positive $f_{\mathrm{NL}}, \beta$ should be negative for the quadratic potential since $A(x)<0$ for the potential. For example, if we take $(\alpha, \beta)=(0.5,-1)$ and assume the quadratic potential and the Yukawa interaction for the inflaton in which $A(x) \sim-1 / 6$, then we have $f_{\mathrm{NL}}=45$ for $R \gg 1$. Since the constraint on $f_{\mathrm{NL}}$ from WMAP 5-year data is given as $-9<f_{\mathrm{NL}}<111$ [7], this sort of possibilities may be interesting. Furthermore this demonstrates that the non-linearity between $\Gamma$ and $\sigma$ can provide non-Gaussianity of $\zeta$ much larger than that from the non-linearity between $\zeta$ and $\delta \Gamma$.

Meanwhile, some symmetries may forbid the appearance of the linear terms in $\sigma$ in $y_{a}(\sigma)$. In this case, Eq. (32) becomes $\simeq M^{2} /\left(2 \beta \sigma^{2}\right)$, where $f_{\mathrm{NL}}$ can be very large because of $M / \sigma \gg 1$ when $\beta<0$. For an illustrational purpose, we will consider two cases: $(\alpha, \beta)=(0.3,-1.0)$ (case $\mathrm{A})$ and $(\alpha, \beta)=(0.0,-1.0)$ (case $\mathrm{B})$ in the following.

To compare the prediction for the primordial curvature fluctuations and non-Gaussianity with observations, we need to specify when the present cosmological scale exited the horizon during inflation. Since $k_{*}=a\left(t_{*}\right) H_{*}$ holds when the scale with the wave number $k_{*}$ crossed the horizon, the reference scale $k_{\text {ref }}$ where we probe the primordial fluctuations at the present time is related to that at the horizon crossing during inflation as

$$
\frac{k_{\mathrm{ref}}}{a_{0} H_{0}}=\frac{a\left(t_{*}\right) H_{*}}{a_{0} H_{0}}
$$

\#2 Here we assume that there are no renormalizable interactions between $\sigma$ and $\psi_{a}, \chi_{a}$ because such interactions will give the modulus a thermal mass much larger than the Hubble parameter, which suppresses fluctuations of modulus and spoils the modulated reheating scenario. 
where $a_{0}$ and $H_{0}$ are the scale factor and the Hubble parameter at present. The ratio of $a_{*}$ to $a_{0}$ on the right-hand side can be divided into several parts as,

$$
\frac{k_{\mathrm{ref}}}{a_{0} H_{0}}=\frac{a\left(t_{*}\right)}{a\left(t_{\mathrm{end}}\right)} \frac{a\left(t_{\mathrm{end}}\right)}{a\left(t_{f}\right)} \frac{a\left(t_{f}\right)}{a_{0}} \frac{H_{*}}{H_{0}} .
$$

The definition of $t_{\text {end }}$ and $t_{f}$ is given in section 3. By taking logarithm of both sides and using Eqs. (6) and (7), the number of $e$-folding between the time $t_{*}$ and $t_{\text {end }}$, i.e., $N\left(t_{\mathrm{end}}, t_{*}, \phi_{*}\right)$ can be written as

$$
N\left(t_{\mathrm{end}}, t_{*}, \phi_{*}\right)=-\log \frac{k_{\mathrm{ref}}}{a_{0} H_{0}}-N\left(t_{c}, t_{\mathrm{end}}\right)-\frac{1}{4} \log \frac{\rho_{c}}{\rho_{f}}-Q(x)+\log \frac{a_{f}}{a_{0}}+\log \frac{H_{*}}{H_{0}},
$$

with which we can determine the field value $\phi_{*}$ at the horizon crossing.

For the reference scale, we take $k_{\text {ref }}=0.002 \mathrm{Mpc}^{-1}$ in the analysis. As for the fifth term, we assume that no more entropy is produced after the inflaton decays. Thus this term can be rewritten by using the conservation of the entropy density per comoving volume. Since the entropy density is given by $s=(2 \pi / 45) g_{* s} T^{3}$ with $g_{* s}$ being the total number of effective massless degrees of freedom, we have the following relation,

$$
\log \frac{a_{f}}{a_{0}}=\frac{1}{3} \log \frac{s_{0}}{s_{f}}=\frac{1}{3} \log \frac{g_{* s 0} T_{0}^{3}}{g_{* s f} T_{f}^{3}} .
$$

For $g_{* s f}$ at the time of $a_{f}$, we take $g_{* s f}=100$. With regard to other quantities, we assume $H\left(t_{c}\right)=10^{-2} H_{\text {end }}$ and $x=10^{-8}$ in the following analyses.

\subsection{Chaotic inflation : $V(\phi)=\frac{m^{2}}{2} \phi^{2}$}

Now let us first consider chaotic inflation [28] with the quadratic potentia\#. Since we have fixed the values of the quantities which determine the background evolution after the end of inflation as mentioned above, remaining variables that we need to specify are some parameters in the inflaton potential and the values of $\sigma$ and $M$ relevant to fluctuations from modulated reheating, which appear in the decay rate of the inflaton. Since the inflation is assumed to be driven solely by the inflaton, the Hubble parameter during inflation is controlled by the value of the parameters in the potential. Thus the primordial curvature fluctuations also depend on these parameters, which means that the parameters in the inflaton potential can be fixed by the WMAP normalization, i.e. by requiring that the total curvature fluctuations are $\mathcal{O}\left(10^{-5}\right)$. In fact, the normalization slightly depends on the spectral index and tensor-to-scalar ratio. Hence we used the one given in Ref. [36] derived using the WMAP 5-year data in the following analyses to fix a

\#3 This type of a simple polynomial potential can be realized in supergravity [29-35].

\#4 The amplitude at $k=0.002 \mathrm{Mpc}^{-1}$ is given with $\delta_{H}^{2}=(4 / 25) \mathcal{P}_{\zeta}$ as

$$
10^{5} \delta_{H}^{\mathrm{WMAP} 5}=1.910 \times \frac{\exp \left[(-0.724+0.533 r)\left(1-n_{s}\right)\right]}{\sqrt{1+0.278 r}} .
$$

For the details, see Ref. [36]. 


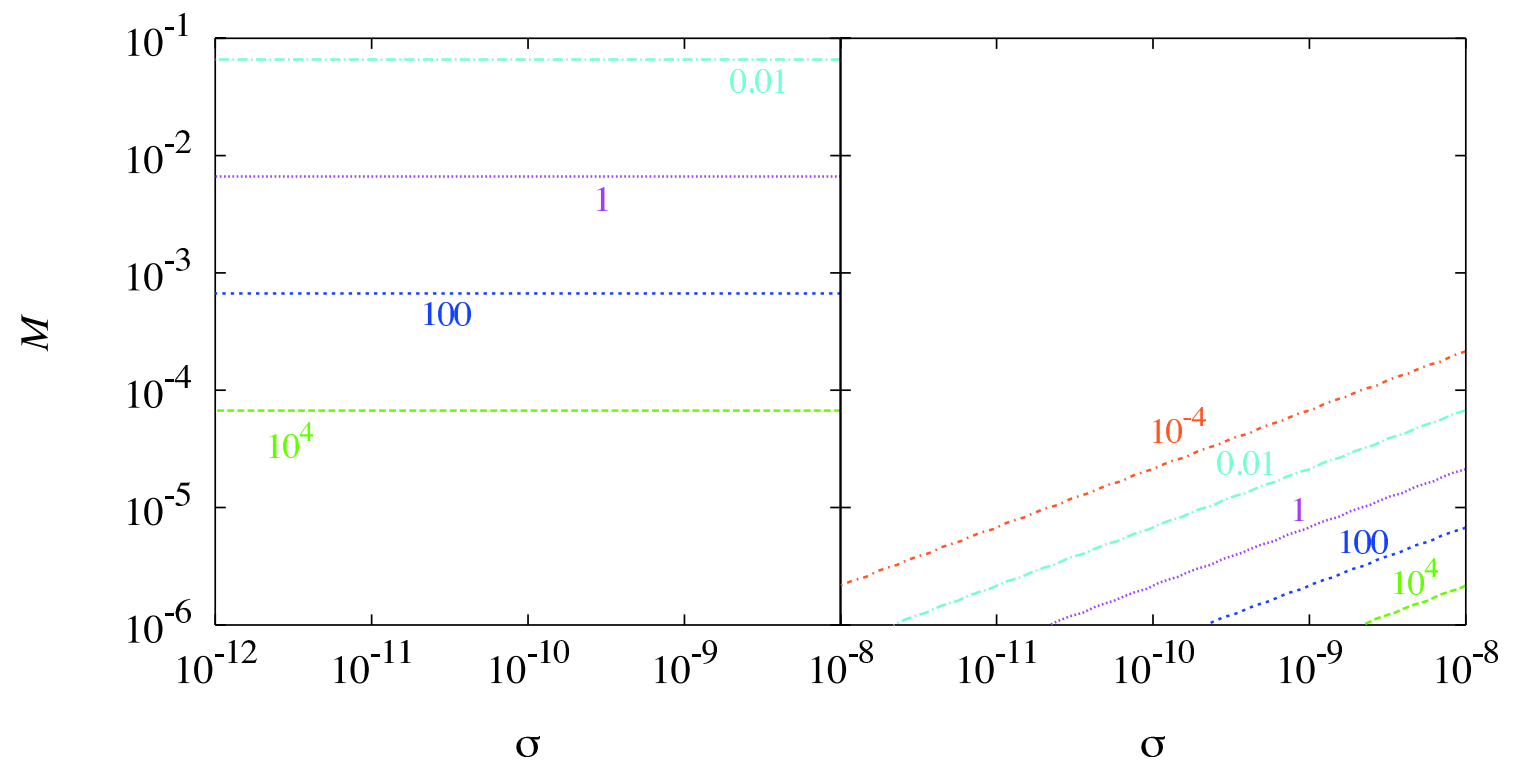

Figure 3: Contours of $R$ in the $\sigma-M$ plane for the chaotic inflation model with quadratic potential. Left and right panels are for case $\mathrm{A}(\alpha=0.3, \beta=-1)$ and case $\mathrm{B}(\alpha=0, \beta=$ -1 ), respectively. In the figure, $M$ and $\sigma$ are shown in units of $M_{\mathrm{pl}}$.

parameter in the potential for the inflaton. For the quadratic case of the chaotic inflation, we fix the value of $m$ by the WMAP normalization. With regard to the spectral index $n_{s}$ and tensor-to-scalar ratio $r$, the observation of WMAP 5-year also give severe constraints on these quantities (see Fig. 3 in Ref. [7]). When fluctuations from the inflaton alone are responsible for the curvature fluctuations, in our setting (fixing the parameters) where the number of $e$-folding during inflation is $N_{\mathrm{inf}} \simeq 60$, these quantities are given as $n_{s}=0.967$ and $r=0.133$ which are allowed by WMAP 5 -year data. However, if fluctuations from the modulus fluctuations are included, these predictions can be modified, which we will discuss in the following. To see in what cases such modifications are significant, we show the contours of $R$ in the $\sigma-M$ plane in Fig. [3 for the case A (left panel) and B (right panel). By looking at the figure, we can expect the parameter region where the predictions for $n_{s}$ and $r$ are modified much.

In the top panels of Fig. 4 , we show contours of the scalar spectral index $n_{s}$ in the $\sigma-M$ plane for the case A (left panel) and B (right panel). Let us first look at the left panel (case A). In this case, $\Gamma_{\sigma} / \Gamma$ is almost independent of $\sigma$. Hence $R$ is also independent of $\sigma$ and depends only on $M$. As can be seen from Eq. (27), the effect of fluctuations from modulated reheating on $n_{s}$ appears only through the parameter $R$. This is the reason why the contours are parallel to the $\sigma$-axis. Furthermore, since $R \propto M^{-2}$, smaller value of $M$ indicates larger $R$, i.e. larger contribution from the modulus fluctuations to the total curvature perturbations. When the modulus contributions become dominant, the value of

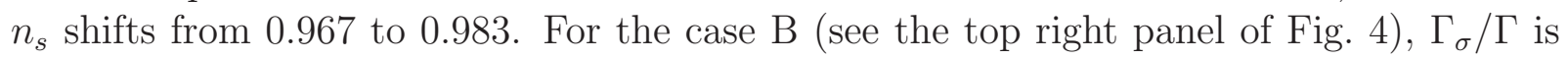




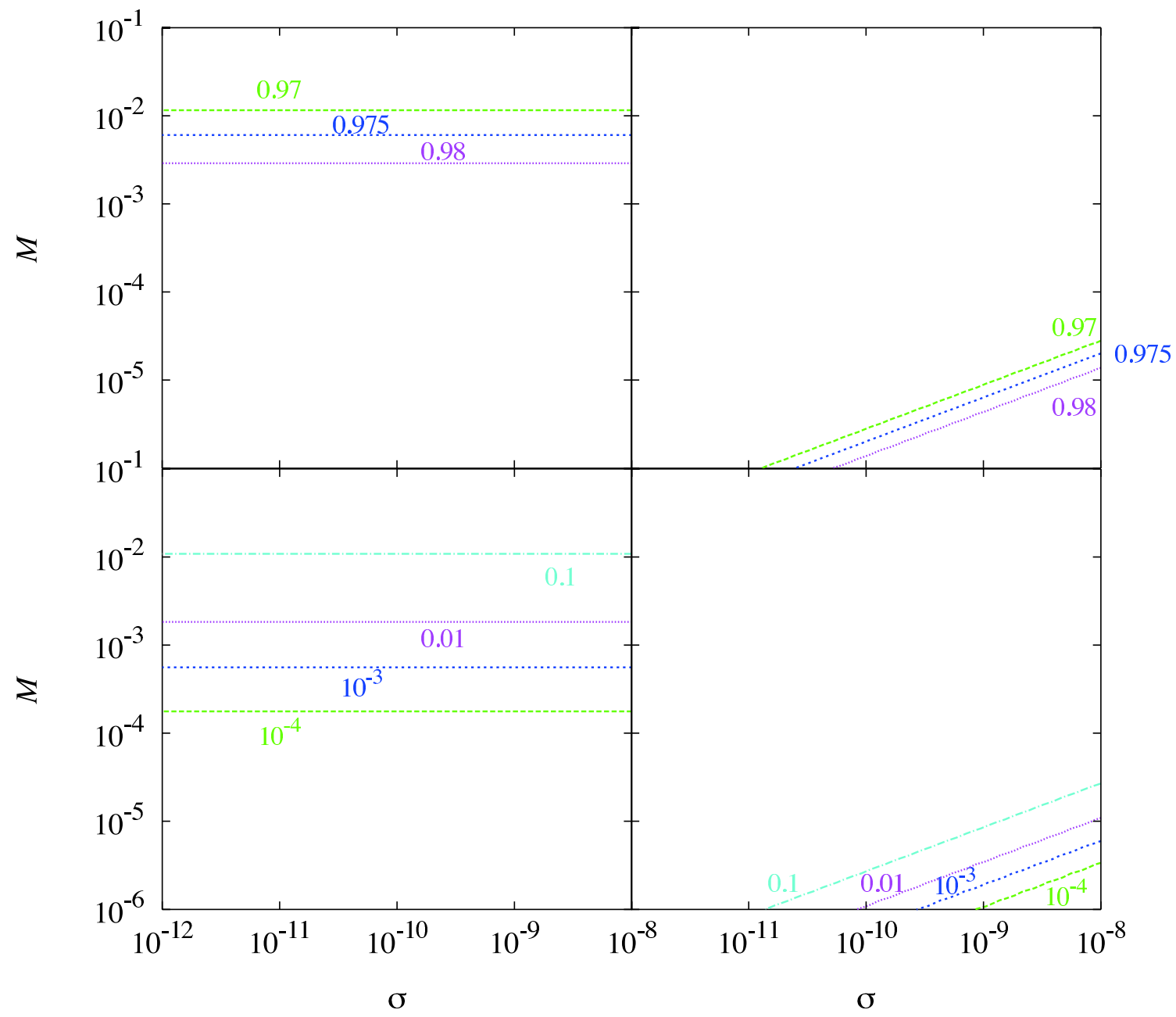

Figure 4: Contours of $n_{s}$ (top panels) and $r$ (bottom panels) in the $\sigma-M$ plane for the chaotic inflation model with quadratic potential. Left (right) panels are for case A (B).

proportional to $\sigma / M^{2}$. Hence the contours are parallel to the line $M / \sigma^{1 / 2}=$ const., which can be seen from the figure.

Now we investigate how the tensor-to-scalar ratio is modified in the mixed model. In

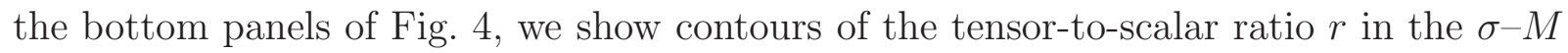
plane. Notice that as in the case of $n_{s}$, the effect of the modulus on $r$ appears only through $R$. Hence the slope of the contours becomes the same as those for $n_{s}$. We see that when the modulus contributions are dominant, $r$ becomes negligibly small.

Next we move on to the issues of non-Gaussianity. For this purpose, we show contours of non-linearity parameters $f_{\mathrm{NL}}, \tau_{\mathrm{NL}}$ and $g_{\mathrm{NL}}$ in the top, middle and bottom panels of Fig. 5, respectively. Remember that, when the fluctuations from the inflaton alone are assumed, these non-linearity parameters are of the orders of the slow-roll parameters, which 


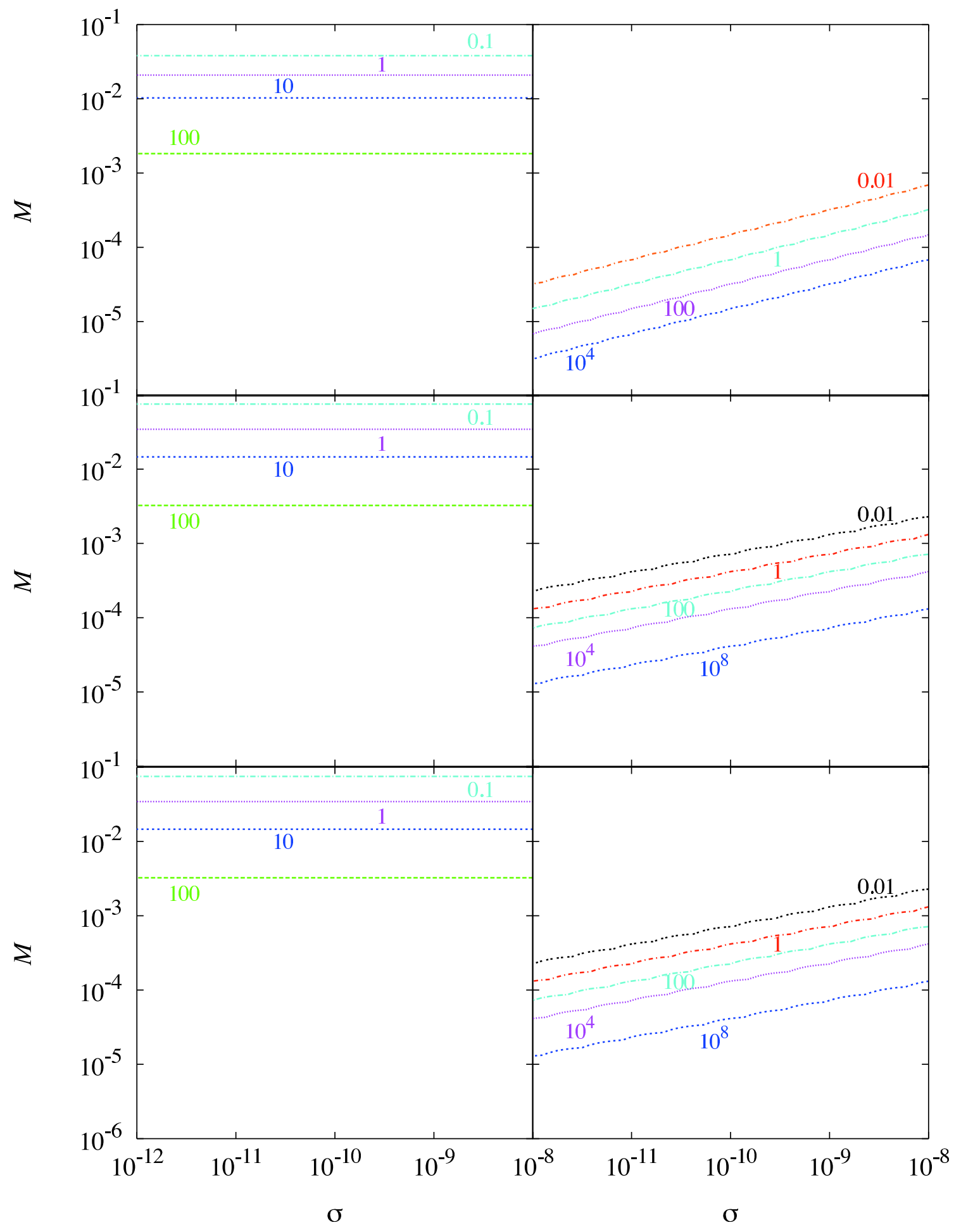

Figure 5: Contours of $f_{\mathrm{NL}}$ in the $\sigma-M$ plane for the chaotic inflation model with quadratic potential. Left (right) panels are for case A (B). 
are usually much less than unity. However, since fluctuations from modulated reheating can give large non-Gaussianity, the non-linearity parameters can be large even when the contribution from the modulus fluctuations is subdominant in the curvature fluctuations. For the case A, we have $\Gamma \Gamma_{\sigma \sigma} / \Gamma_{\sigma}^{2} \simeq$ const. (see Eq. (32)). Hence as in the cases for $n_{s}$ and $r$, the value of $R$ alone determines the effects of the modulus contributions on the non-linearity parameters. Thus the smaller $M$ gives the larger non-Gaussianity which can be seen from the figures. On the other hand, for the case B, the above mentioned combination is $\Gamma \Gamma_{\sigma \sigma} / \Gamma_{\sigma}^{2} \simeq M^{2} / \sigma^{2}$. Hence $\sigma$ and $R$ can both affect the non-linearity parameters in this case. If the former effect is dominant, the slope of the contour becomes $1 / 2$. On the other hand, if the latter one is dominant, its slope becomes 1 and we see that for larger $R$ and smaller $M^{2} / \sigma^{2}$, we have larger non-Gaussianity. In fact, in Fig. 5 , contours corresponding to the latter case are not shown for this model, since the size of the non-linearity parameters become too large in such a region for this model, thus we do not depict them here. However this kind of behavior can be seen in Figs. 10 and 12 which are for other inflation models to be discussed in the following.

For an illustrational purpose, we fix the values of $\alpha$ and $\beta$. However, the predictions for the inflationary parameters also depends on these values. Thus here we stop to discuss its dependence. In Fig. 6, contours of the ratio $R$ are shown in the $\alpha-M$ plane. Since the relative size between $\alpha$ and $\beta$ is important to see the effects of the modulated reheating, here we fix the value of $\beta$ and vary $\alpha$. Since the ratio $R$ is controlled by the combination $\Gamma_{\sigma}^{2} / \Gamma^{2}$, as $\alpha$ increases, $R$ also becomes large for $M$ being fixed. As discussed in the previous section, $n_{s}$ and $r$ are also determined by the above combination, thus the tendencies are the same as that of the ratio $R$, which can be seen in Fig. (7) where contours of $n_{s}$ and $r$ are depicted.

However, if we look at plots of non-linearity parameters, which are shown in Fig. 8, the trends are different. As mentioned before, the non-linearity parameters are governed by the combination $\Gamma \Gamma_{\sigma \sigma} / \Gamma_{\sigma}^{2} \simeq 2 \beta /(\alpha+2 \beta \sigma / M)^{2}$, when $\alpha$ is small, the dependence of non-linearity parameters on $\alpha$ is also small. On the other hand, when $\alpha$ is large, the size of the above combination is determined by $\alpha$ with $\beta$ being fixed. Thus the predictions are irrelevant to $M$ in this case, which can be read off from the figure. In the following, we discuss other types of the inflaton potential and again present our results in the $\sigma-M$ plane fixing the values of $\alpha$. However, the trend discussed here also applies to those cases.

\subsection{Chaotic inflation : $V(\phi)=\frac{\lambda}{4} \phi^{4}+\frac{m^{2}}{2} \phi^{2}$}

Next we consider chaotic inflation with the quartic potential. As a simple possibility, one usually assumes that the quartic term alone exists in the potential for the inflaton. Since the energy density of inflaton oscillations in such a potential decreases in the same way as that of radiation, no fluctuation is generated via modulated reheating scenario as discussed previously. However, there are some interesting models $[37,38]$ where the potential during inflation is given by the quartic form, but the quadratic term becomes effective when the inflaton starts to oscillate. In fact, this feature comes from the curved trajectory 


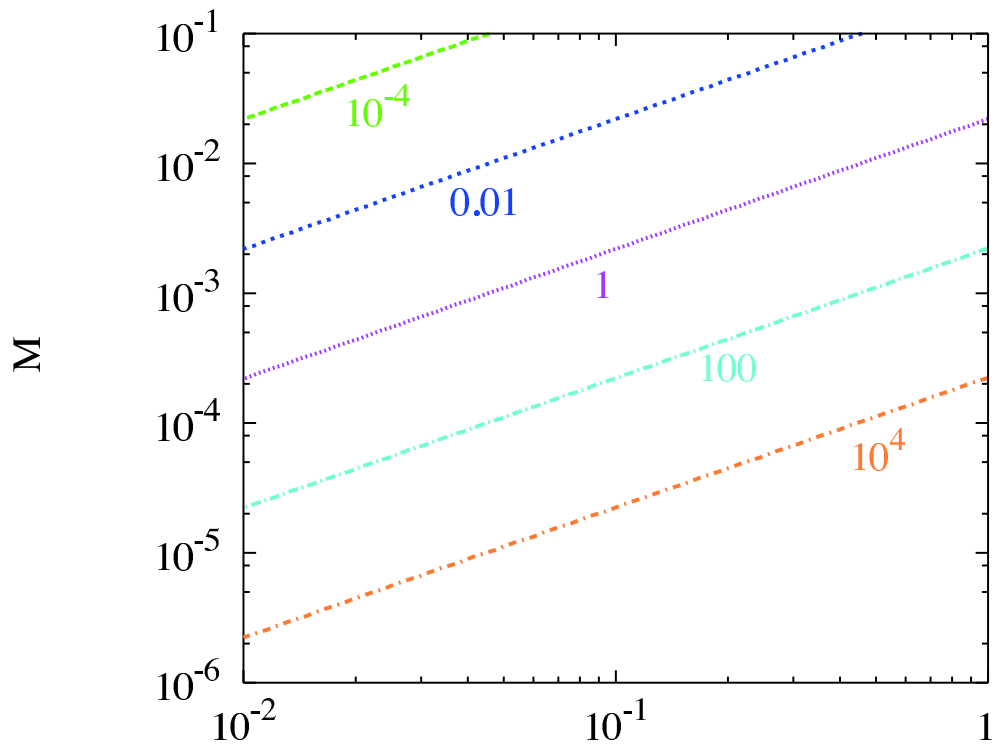

$\alpha$

Figure 6: Contours of $R$ in the $\alpha-M$ plane for the chaotic inflation model with a quadratic potential.

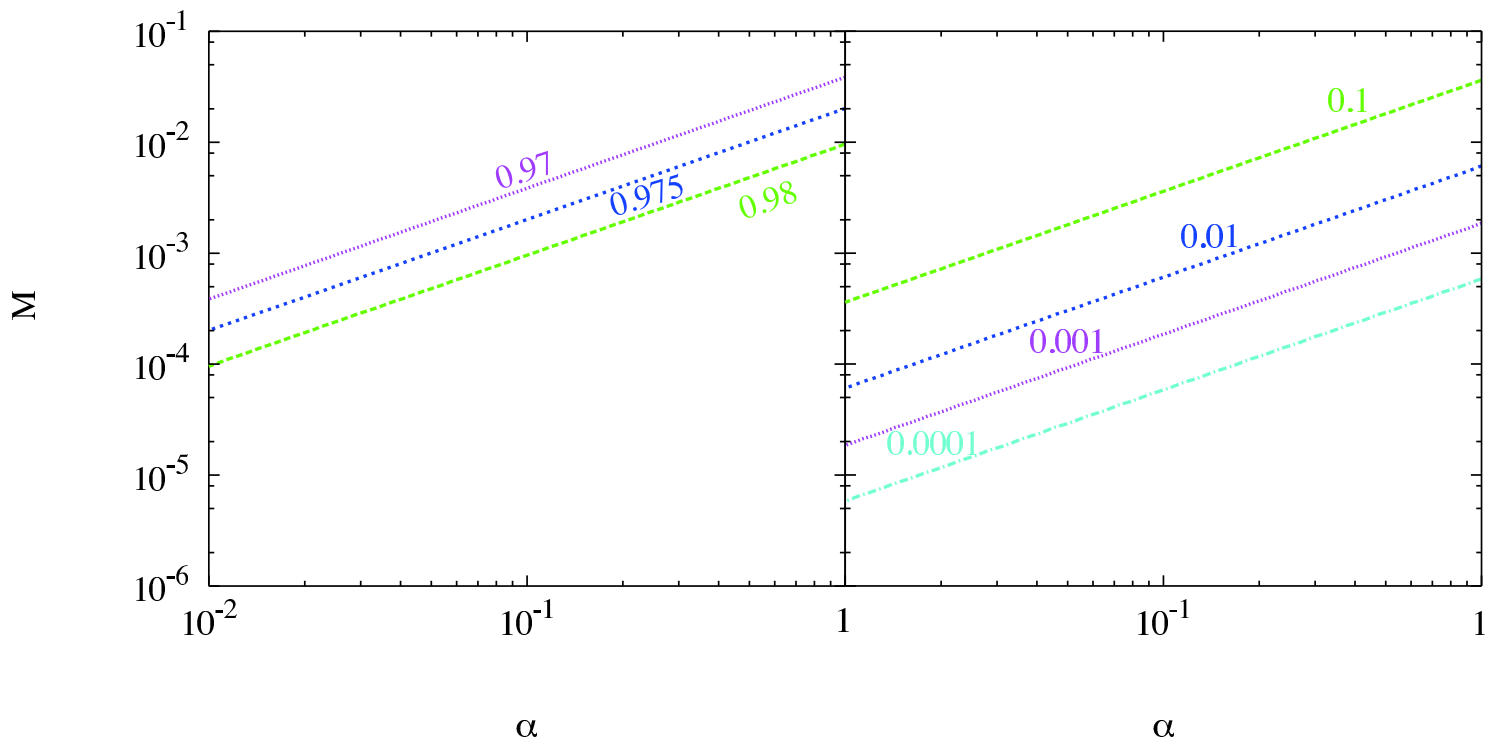

Figure 7: Contours of $n_{s}$ (left panel) and $r$ (right panel) in the $\alpha-M$ plane for the chaotic inflation model with a quadratic potential. 


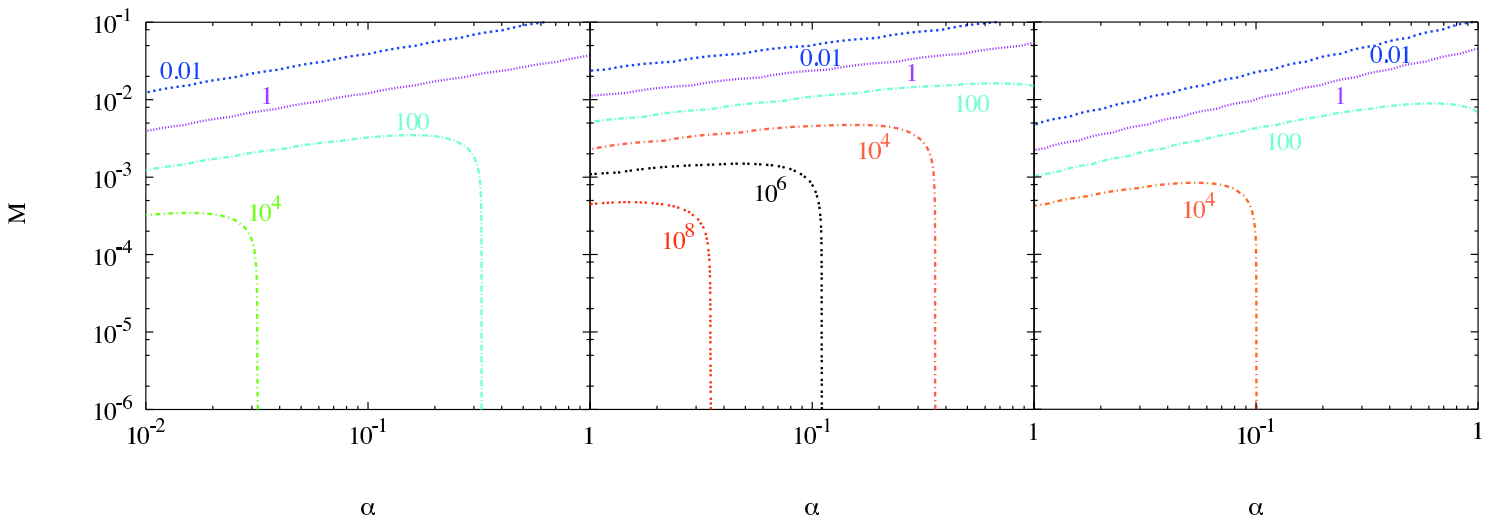

Figure 8: Contours of $f_{\mathrm{NL}}$ (left), $\tau_{\mathrm{NL}}$ (center) and $g_{\mathrm{NL}}$ (right) in the $\alpha-M$ plane for the chaotic inflation model with a quadratic potential.

in the multiple fields configuration. But it can be well characterized by considering a single field with the potential given by the sum of the quadratic term and the quartic one: $V(\phi)=\frac{m^{2}}{2} \phi^{2}+\frac{\lambda}{4} \phi^{4}$. Above situation can be realized by taking $m=\sqrt{\lambda} M_{\mathrm{pl}}$ with $\lambda$ being fixed by WMAP normalization. Hence we adopt this relation in the following analysis for definiteness. In this case, quartic term dominates during inflation $\left(\phi \gtrsim M_{\mathrm{pl}}\right)$, but the quadratic one dominates after inflation.

This model is also interesting in another aspect. In the absence of the quadratic term, we have $Q=0$ and the curvature perturbations are not generated by the modulation of the decay rate. Then the curvature perturbations originate solely from the inflaton fluctuations even when the decay rate fluctuates, which is completely ruled out from WMAP 5-year data because of the too large tensor-to-scalar ratio. However, if we include the quadratic one, the curvature perturbations can be additionally generated when inflaton decays. If these perturbations dominate the total curvature perturbations, the quartic inflation model may still satisfy the observational constraints, with large amount of the non-Gaussianity which could be tested by the future observations.

Fig. 9 show contour plots of $n_{s}$ and $r$ in this case, respectively. Shaded regions are excluded by the WMAP 5-year constraints on $\left(n_{s}, r\right)$. We see that when the modulus contributions become dominant, $n_{s}$ gets closer to unity and $r$ becomes very small. As a result, $\left(n_{s}, r\right)$ enters the allowed region of WMAP 5-year data. The slopes of the contours are essentially the same as in the previous case where $V(\phi)=\frac{m^{2}}{2} \phi^{2}$.

In Fig. 10, we show the contour plots of the three non-linearity parameters. As for the case $\mathrm{A}$, there is a large parameter space where $\left(n_{s}, r\right)$ is in the allowed region of WMAP 5 -year data while generating large amount of non-Gaussianity $f_{\mathrm{NL}}=\mathcal{O}(10 \sim 100)$ which could be tested by the upcoming observations. 


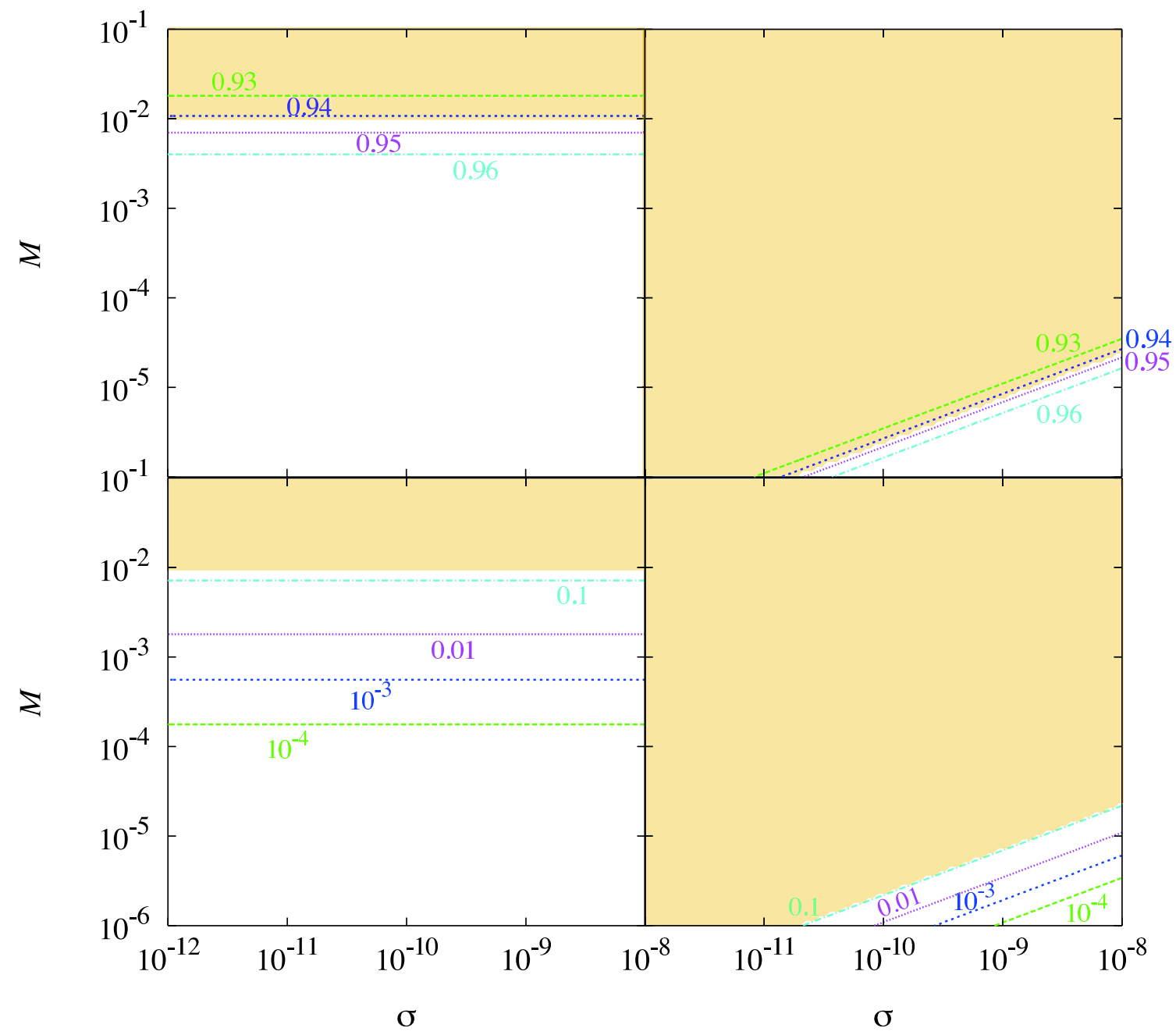

Figure 9: Contours of $n_{s}$ (top panels) and $r$ (bottom panels) in the $\sigma-M$ plane for the chaotic inflation model with quadratic and quartic potential. Left (right) panels are for case A (B). Shaded region is excluded by WMAP5.

5.3 Chaotic inflation : $V(\phi)=\frac{V_{0}}{6}\left(\frac{\phi}{M_{\mathrm{pl}}}\right)^{6}$

Let us next consider chaotic inflation model whose potential is given by

$$
V(\phi)=\frac{V_{0}}{6}\left(\frac{\phi}{M_{\mathrm{pl}}}\right)^{6} .
$$

This model is also completely ruled out by WMAP 5-year data if the curvature perturbations solely originate from the inflaton fluctuations.

Fig. 11 show the contour plots of $n_{s}$ and $r$ in this case, respectively. We see that as in the case of the quadratic and quartic potential, $\left(n_{s}, r\right)$ enters the allowed region of WMAP 


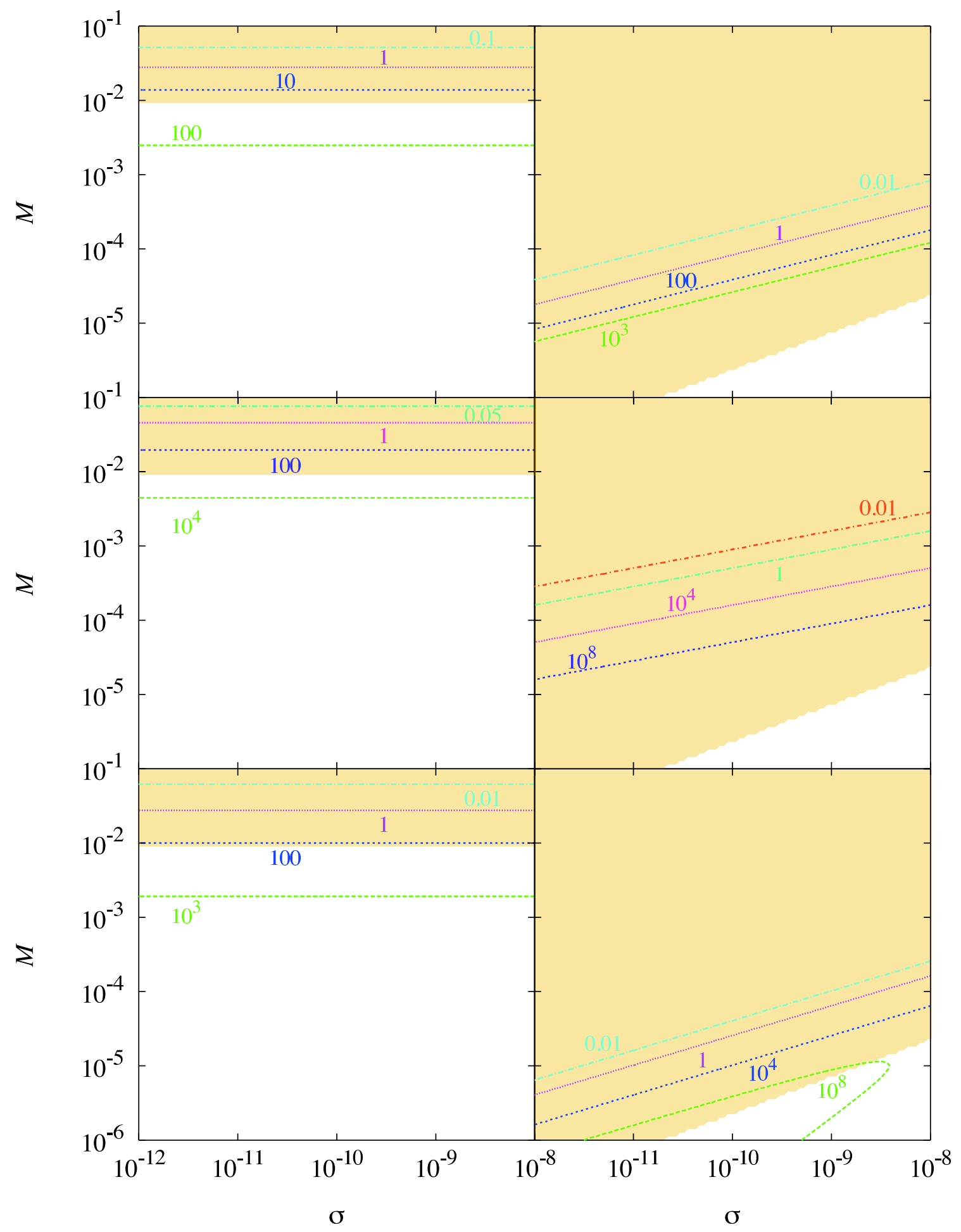

Figure 10: Contours of $f_{\mathrm{NL}}$ (top panels), $\tau_{\mathrm{NL}}$ (middle panels) and $g_{\mathrm{NL}}$ (bottom panels) in the $\sigma-M$ plane for the chaotic inflation model with quadratic and quartic potential. Left (right) panels are for case A (B). Shaded region is excluded by WMAP5. 


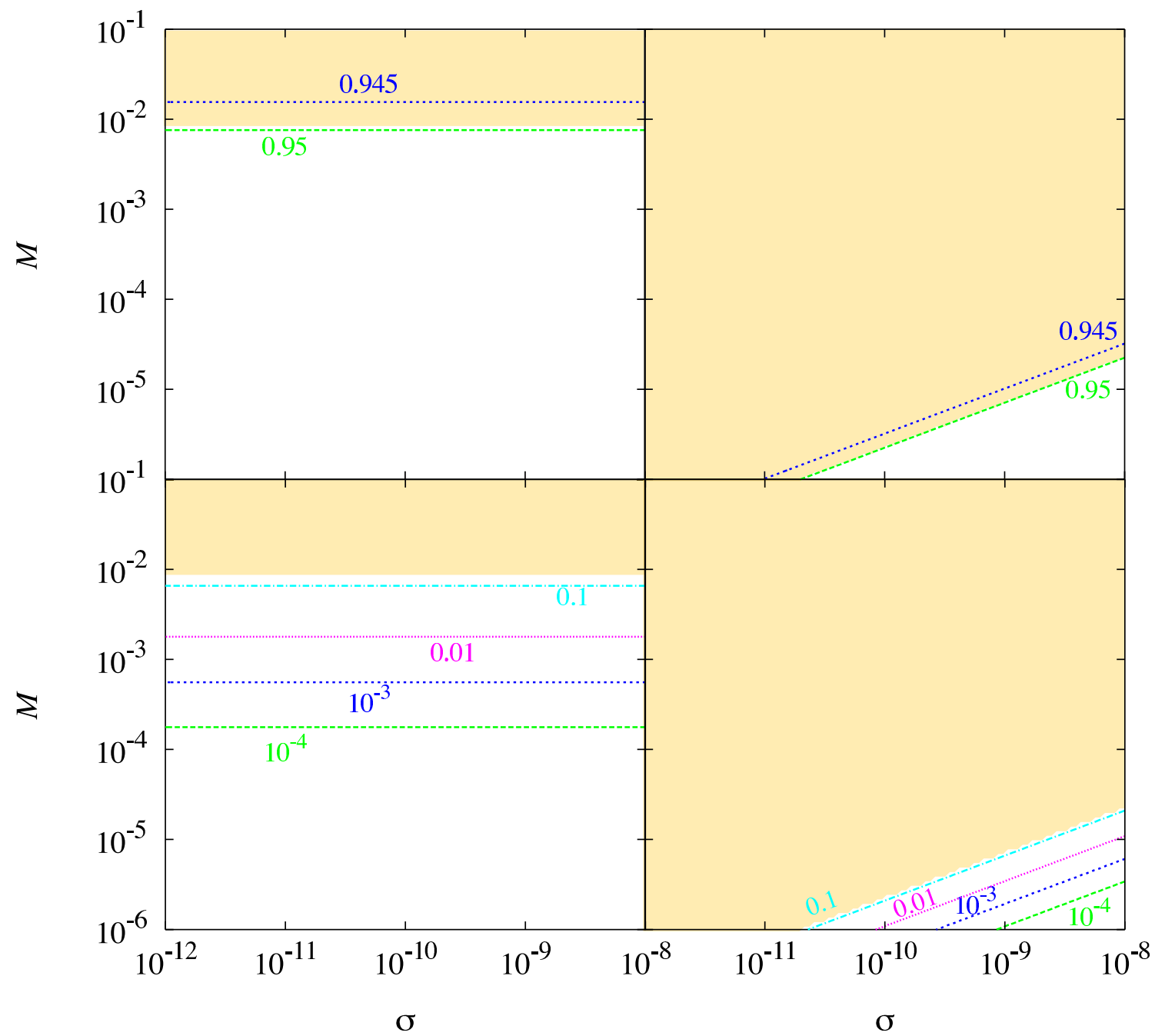

Figure 11: Contours of $n_{s}$ (top panels) and $r$ (bottom panels) in the $\sigma-M$ plane for the chaotic inflation model with sextic potential. Left (right) panels are for case A (B). Shaded region is excluded by WMAP5.

5-year data due to the slight shift of $n_{s}$ to unity and the significant suppression of $r$ when the modulus contributions become dominant.

In Fig. 12, we show the contour plots of the three non-linearity parameters. For the sextic potential, $f_{\mathrm{NL}}$ becomes negatively large when the modulus contributions are dominant, as discussed in the previous section. If we take $\beta<0$ instead of $\beta>0$, then we have positively large $f_{\mathrm{NL}}$ just like the case in the quadratic and quartic potential. 


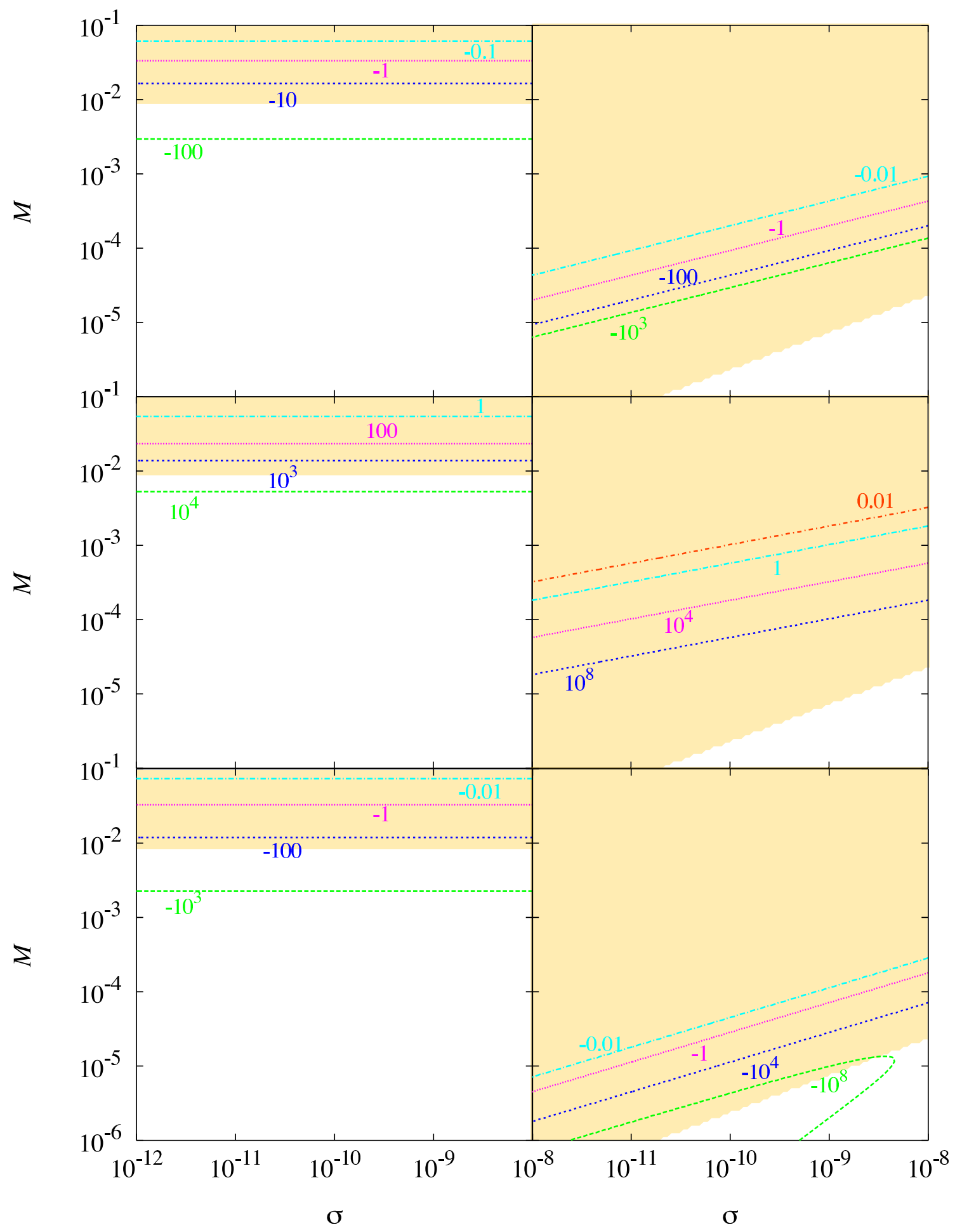

Figure 12: Contours of $f_{\mathrm{NL}}$ (top panels), $\tau_{\mathrm{NL}}$ (middle panels) and $g_{\mathrm{NL}}$ (bottom panels) in the $\sigma-M$ plane for the chaotic inflation model with sextic potential. Left (right) panels are for case A (B). Shaded region is excluded by WMAP5. 


\section{Summary}

We have investigated a mixed scenario where both fluctuations from the inflaton and modulated reheating are responsible for cosmic density fluctuations today. First we summarized the decay rate of the inflaton for various interactions and inflaton potentials, whose details are presented in Appendix A. Then we gave expressions for the spectral index, tensor-to-scalar ratio and non-linearity parameters such as $f_{\mathrm{NL}}, \tau_{\mathrm{NL}}$ and $g_{\mathrm{NL}}$. We found that while the non-linearity between $\zeta$ and $\delta \Gamma$ generates $f_{\mathrm{NL}}=\mathcal{O}(1 \sim 10)$, the nonlinearity between $\Gamma$ and the modulus can generate large $f_{\mathrm{NL}} \geq \mathcal{O}(100)$. We also derived the consistency relation Eq. (25) among the non-linearity parameters which is applicable for the cases where the decay rate for the inflaton is given as Eq. (31). If the potential around the minimum is quadratic, then $g_{\mathrm{NL}}$ takes the same sign as $f_{\mathrm{NL}}$. This is in sharp contrast to the situation where the mixed model of the inflaton and the curvaton yields a negative $g_{\mathrm{NL}}$ when $f_{\mathrm{NL}}$ is large positive.

We have also studied the inflationary parameters including non-linearity parameters assuming some inflation models. Since the new inflation model usually gives a extremely small value of $\epsilon$, we have $R \ll 1$. Thus, the addition of fluctuations from modulated reheating does not significantly change the prediction of the property of fluctuations in the case of new inflation. Therefore, in this paper, we have considered chaotic inflation models with several polynomials and some types of decay rate for an illustrational purpose. In the case with the chaotic inflation model, the contribution from fluctuations of modulated reheating makes non-Gaussianity larger, the spectral index closer to scale-invariant and tensor-to-scalar ratio more suppressed. This helps to liberate models of inflation such as the sextic potential one because this model has been excluded by the data because of its too red-tilted spectrum and too large tensor-to-scalar ratio. The chaotic inflation with quartic potential has also in fact been excluded by the data. Since fluctuations are not generated by modulated reheating in a simple quartic potential case, this model cannot be made viable by just adding a contribution from the modulated reheating. However, even if the inflaton is driven by a quartic term, another term such as a quadratic one can become effective during reheating stage after inflation. This kind of situation can be realized by assuming $V(\phi)=(\lambda / 4) \phi^{4}+(1 / 2) m^{2} \phi^{2}$ with $m=\sqrt{\lambda} M_{\mathrm{pl}}$, which was discussed in this paper. In such a case, fluctuations from modulated reheating can affect the total fluctuations and liberate the model. In addition, non-Gaussianity can also be large in this case too. We have also investigated the case with the quadratic inflation model and showed that non-Gaussianity can be large in this model by just adding some contribution from modulated reheating without conflicting the observations of the spectral index and tensor modes.

In most works so far, observational consequences for various generation mechanisms of primordial fluctuations have been discussed in each separately. However, different mechanisms can be in general responsible for today's cosmic density fluctuations simultaneously so it is of great importance to investigate a mixed model such as the one we discussed in this paper. Precise cosmological observations expected in the near future to hunt for 
primordial non-Gaussianity may reveal such interesting scenarios in the early universe.

Acknowledgments: T.S. is grateful to Masahiro Kawasaki and Fuminobu Takahashi for helpful discussion. T.S. also thanks to the computer system at the Yukawa Institute for Theoretical Physics, Kyoto University, for the numerical calculations. This work is supported in part by the JSPS Postdoctoral Fellowship for Research Abroad (K.I.), the Sumitomo Foundation (T.T.), and the Grant-in-Aid for Scientific Research from the Ministry of Education, Science, Sports, and Culture of Japan No. 19740145 (T.T.), No. 18740157, and No. 19340054 (M.Y.). 


\section{Appendix}

\section{A Calculation of the decay rate}

In this appendix, we calculate the decay rate of the inflaton to lighter particles for three different types of interactions [39-41]. We also consider three different types of potentials around the minimum: quadratic, quartic and sextic potentials. In the following, we approximate the background space-time as Minkowski space because the energies of created particles from the inflaton are much higher than the Hubble parameter. In this appendix, we follow the notations of Ref. [42].

\section{A.1 Yukawa interaction: $\mathcal{L}_{\text {int }}=-y \phi \bar{\psi} \psi$}

We regard $\phi$ as the classical field which is spatially homogeneous but oscillates in time $t$. Then the interaction part of the Hamiltonian is given by

$$
\hat{V}=y \phi(t) \int d^{3} x \bar{\psi} \psi
$$

$\psi$ in the interaction picture is equal to that in the Heisenberg picture when the interaction is absent. Hence we write $\psi$ as

$$
\psi(x)=\sum_{\sigma} \int \frac{d^{3} p}{(2 \pi)^{3 / 2}}\left(u(\vec{p}, \sigma) e^{i p x} b_{\vec{p}, \sigma}+v(\vec{p}, \sigma) e^{-i p x} b_{\vec{p}, \sigma}^{c \dagger}\right),
$$

where $\sigma$ denotes the helicity and $b_{\vec{p}, \sigma}$ and $b_{\vec{p}, \sigma}^{c}$ satisfy the following relations,

$$
\left\{b_{\vec{p}, \sigma}, b_{\vec{q}, \sigma^{\prime}}^{\dagger}\right\}=\left\{b_{\vec{p}, \sigma}^{c}, b_{\vec{q}, \sigma^{\prime}}^{c \dagger}\right\}=\delta(\vec{p}-\vec{q}) \delta_{\sigma \sigma^{\prime}}, \quad\left\{b_{\vec{p}, \sigma}, b_{\vec{q}, \sigma^{\prime}}\right\}=\left\{b_{\vec{p}, \sigma}^{c}, b_{\vec{q}, \sigma^{\prime}}^{c}\right\}=0 .
$$

Also, $u(\vec{p}, \sigma)$ and $v(\vec{p}, \sigma)$ satisfy the following equations,

$$
\left(i p^{\mu} \gamma_{\mu}+m_{\psi}\right) u(\vec{p}, \sigma)=0, \quad\left(-i p^{\mu} \gamma_{\mu}+m_{\psi}\right) v(\vec{p}, \sigma)=0 .
$$

Let us write the period of the oscillations of $\phi(t)$ as $T$ and Fourier expand $\phi(t)$ as

$$
\phi(t)=\sum_{n=-\infty}^{\infty} \phi_{n} e^{-i \omega n t}
$$

where $\omega \equiv 2 \pi / T$.

Using these, the transition amplitude from the initial state $|i\rangle=|0\rangle$ at $t=-\infty$ to the final two-particle state $|f\rangle=b_{\vec{p}, \sigma}^{\dagger} b_{\vec{q}, \sigma^{\prime}}^{c \dagger}|0\rangle$ at $t=\infty$ is given by

$$
-i \int_{-\infty}^{\infty} d t\langle f|V(t)| i\rangle=-2 \pi i y \delta(\vec{p}+\vec{q}) \bar{u}(\vec{p}, \sigma) v\left(-\vec{p}, \sigma^{\prime}\right) \sum_{n=-\infty}^{\infty} \phi_{n} \delta\left(2 E_{p}-n \omega\right)
$$


where $E_{p} \equiv \sqrt{p^{2}+m_{\psi}^{2}}$ is the energy of the $\psi$ particle. Then $\Gamma$, the transition rate per unit time and unit volume, is given by

$$
\begin{aligned}
\Gamma & =y^{2} \sum_{n=1}^{\infty}\left|\phi_{n}\right|^{2} \int \frac{d^{3} p}{(2 \pi)^{2}} \delta\left(2 E_{p}-n \omega\right) \sum_{\sigma \sigma^{\prime}}\left|\bar{u}(\vec{p}, \sigma) v\left(-\vec{p}, \sigma^{\prime}\right)\right|^{2} \\
& =\frac{y^{2}}{4 \pi} \omega^{2} \sum_{n=1}^{\infty} n^{2}\left|\phi_{n}\right|^{2} \\
& =\frac{y^{2}}{8 \pi}\left\langle\dot{\phi}^{2}\right\rangle .
\end{aligned}
$$

Here $\langle\cdots\rangle$ denotes the average of $\cdots$ over one period of the oscillations. From the first line to the second one, we have used the relation,

$$
\sum_{\sigma \sigma^{\prime}}\left|\bar{u}(\vec{p}, \sigma) v\left(-\vec{p}, \sigma^{\prime}\right)\right|^{2}=\frac{2 p^{2}}{E_{p}^{2}}
$$

and assumed $\psi$ is massless, i.e., $m_{\psi}=0$.

$\Gamma$ is the production rate of two $\psi$-particle from the vacuum, not the decay rate of $\phi$-field. The decay rate of the $\phi$ energy, which we denote as $\Gamma_{\phi}$, can be obtained from the energy conservation,

$$
\rho_{\phi} \Gamma_{\phi} \Delta t=E \Gamma \Delta t
$$

The left-hand side denotes the energy loss of the $\phi$-field during the infinitesimal time $\Delta t$ while the right-hand side the energy gain of two $\psi$-particles. $E$ is the expectation value of the energy of the final two-particle state. From this equation, $\Gamma_{\phi}$ can be written as

$$
\Gamma_{\phi}=\frac{y^{2}}{8 \pi} E \frac{\left\langle\dot{\phi}^{2}\right\rangle}{\rho_{\phi}} .
$$

From the definition of $E$, it can be written as

$$
E=\frac{\sum_{n, \sigma, \sigma^{\prime}} \int d^{3} p d^{3} q \delta(\vec{p}+\vec{q}) E_{f} \delta\left(E_{f}-n \omega\right)\left|\mathcal{M}_{n}\right|^{2}}{\sum_{n, \sigma, \sigma^{\prime}} \int d^{3} p d^{3} q \delta(\vec{p}+\vec{q}) \delta\left(E_{f}-n \omega\right)\left|\mathcal{M}_{n}\right|^{2}}
$$

where $E_{f}$ is the energy of the final state and $\mathcal{M}_{n}$ is defined by

$$
\mathcal{M}_{n}=-2 \pi y i \bar{u}(\vec{p}, \sigma) v\left(-\vec{p}, \sigma^{\prime}\right) \phi_{n}
$$

Substituting this into the equation above yields

$$
E=\frac{\sum_{n=1}^{\infty}\left|\phi_{n}\right|^{2}(n \omega)^{3}}{\sum_{n=1}^{\infty}\left|\phi_{n}\right|^{2}(n \omega)^{2}}
$$


Let us define the numerical factor $\alpha$ by

$$
\alpha=\frac{\sum_{n=1}^{\infty}\left|\phi_{n}\right|^{2} n^{3}}{\sum_{n=1}^{\infty}\left|\phi_{n}\right|^{2} n^{2}} .
$$

Then $\Gamma_{\phi}$ can be written as

$$
\Gamma_{\phi}=\frac{y^{2}}{8 \pi} \omega \alpha \frac{\left\langle\dot{\phi}^{2}\right\rangle}{\rho_{\phi}} .
$$

Hence once we specify the motion of $\phi(t)$, we can immediately calculate $\Gamma_{\phi}$ using Eq. (53).

\section{A.1.1 Quadratic potential: $V(\phi)=\frac{m^{2}}{2} \phi^{2}$}

In this case, $\phi(t)$ can be written as

$$
\phi(t)=\phi_{0} \cos (m t),
$$

where the frequency, $m$, is just equal to the mass of the inflaton.

Then, we have

$$
\alpha=1, \quad \frac{\left\langle\dot{\phi}^{2}\right\rangle}{\rho_{\phi}}=1
$$

Hence $\Gamma_{\phi}$ is given by

$$
\Gamma_{\phi}=\frac{y^{2}}{8 \pi} m
$$

\section{A.1.2 Quartic potential: $V(\phi)=\frac{\lambda}{4} \phi^{4}$}

In this case, $\phi(t)$ can be written as

$$
\phi(t)=\frac{\sqrt{\pi} \Gamma\left(\frac{3}{4}\right)}{\Gamma\left(\frac{5}{4}\right)} \phi_{0} \sum_{n=1}^{\infty}\left(e^{i(2 n-1) \omega t}+e^{-i(2 n-1) \omega t}\right) \frac{e^{-\frac{\pi}{2}(2 n-1)}}{1+e^{-\pi(2 n-1)}},
$$

where the frequency $\omega$ is given by

$$
\omega=\frac{1}{2} \sqrt{\frac{\pi}{6}} \frac{\Gamma\left(\frac{3}{4}\right)}{\Gamma\left(\frac{5}{4}\right)} m_{\phi}^{\mathrm{eff}} .
$$

$m_{\phi}^{\text {eff }}$ is the effective mass of $\phi$ defined by

$$
m_{\phi}^{\mathrm{eff}} \equiv \sqrt{3 \lambda} \phi_{0} .
$$


Using these, we find

$$
\alpha \approx 1.036, \quad \frac{\left\langle\dot{\phi}^{2}\right\rangle}{\rho_{\phi}}=\frac{4}{3} .
$$

Then the decay rate of the inflaton can be written as

$$
\Gamma_{\phi}=A_{2} \frac{y^{2}}{8 \pi} m_{\phi}^{\mathrm{eff}},
$$

where $A_{2} \approx 0.676$ is a numerical constant.

\section{A.1.3 Sextic potential: $V(\phi)=\frac{V_{0}}{6 M_{\mathrm{pl}}^{6}} \phi^{6}$}

In this case, the frequency of the inflaton oscillations is given by

$$
\omega=\frac{1}{2} \sqrt{\frac{\pi}{15}} \frac{\Gamma\left(\frac{2}{3}\right)}{\Gamma\left(\frac{7}{6}\right)} m_{\phi}^{\mathrm{eff}} .
$$

$m_{\phi}^{\text {eff }}$ is the effective mass of $\phi$ defined by

$$
m_{\phi}^{\mathrm{e} f} \equiv \frac{\sqrt{5 V_{0}}}{M_{\mathrm{pl}}^{3}} \phi_{0}^{2}
$$

To determine $\alpha$, we numerically solved the equation of motion for $\phi$ over one period of the oscillations. The result is

$$
\alpha \approx 1.0897, \quad \frac{\left\langle\dot{\phi}^{2}\right\rangle}{\rho_{\phi}}=\frac{3}{2} .
$$

Then the decay rate of the inflaton can be written as

$$
\Gamma_{\phi}=A_{3} \frac{y^{2}}{8 \pi} m_{\phi}^{\mathrm{eff}},
$$

where $A_{3} \approx 0.546$ is a numerical constant.

\section{A.2 Interaction with scalar field $\chi: \mathcal{L}_{\text {int }}=-M \phi \chi \chi$}

In this case, the interaction part of the Hamiltonian is given by

$$
\hat{V}=M \phi(t) \int d^{3} x \hat{\chi} \hat{\chi}
$$

Let us expand $\chi$ as

$$
\chi(x)=\int \frac{d^{3} p}{(2 \pi)^{3 / 2} \sqrt{2 E_{p}}}\left(e^{i p x} a_{\vec{p}}+e^{-i p x} a_{\vec{p}}^{\dagger}\right) .
$$


Then the transition amplitude from the initial state $|i\rangle=|0\rangle$ at $t=-\infty$ to the final two-particle state $|f\rangle=a_{\vec{p}}^{\dagger} a_{\vec{q}}^{\dagger}|0\rangle$ is given by

$$
-i \int_{-\infty}^{\infty} d t\langle f|V(t)| i\rangle=-2 \pi i M \delta(\vec{p}+\vec{q}) \sum_{n=-\infty}^{\infty} \frac{\phi_{n}}{E_{p}} \delta\left(2 E_{p}-n \omega\right) .
$$

The transition rate per unit time and unit volume becomes

$$
\begin{aligned}
\Gamma & =\frac{M^{2}}{4 \pi} \sum_{n=1}^{\infty}\left|\phi_{n}\right|^{2} \\
& =\frac{M^{2}}{8 \pi}\left\langle\phi^{2}\right\rangle .
\end{aligned}
$$

The corresponding decay rate of the inflaton is given by

$$
\Gamma_{\phi}=\frac{M^{2}}{8 \pi} E \frac{\left\langle\phi^{2}\right\rangle}{\rho_{\phi}},
$$

where $E$, the mean energy of the two-particle state, is given by

$$
E=\frac{\sum_{n=1}^{\infty} n \omega\left|\phi_{n}\right|^{2}}{\sum_{n=1}^{\infty}\left|\phi_{n}\right|^{2}}
$$

Introducing the dimensionless number $\beta$ by

$$
\beta \equiv \frac{\sum_{n=1}^{\infty} n\left|\phi_{n}\right|^{2}}{\sum_{n=1}^{\infty}\left|\phi_{n}\right|^{2}}
$$

$\Gamma_{\phi}$ becomes

$$
\Gamma_{\phi}=\frac{M^{2}}{8 \pi} \beta \omega \frac{\left\langle\phi^{2}\right\rangle}{\rho_{\phi}} .
$$

\section{A.2.1 Quadratic potential: $V(\phi)=\frac{m^{2}}{2} \phi^{2}$}

Using Eq. (54), we have

$$
\beta=1, \quad \frac{\left\langle\phi^{2}\right\rangle}{\rho_{\phi}}=\frac{1}{m^{2}} .
$$

Hence $\Gamma_{\phi}$ is given by

$$
\Gamma_{\phi}=\frac{M^{2}}{8 \pi m}
$$




\section{A.2.2 Quartic potential: $V(\phi)=\frac{\lambda}{4} \phi^{4}$}

Using Eq. (57), we find

$$
\beta \approx 1.004, \quad \frac{\left\langle\phi^{2}\right\rangle}{\rho_{\phi}} \approx \frac{5.48}{\left(m_{\phi}^{\mathrm{eff}}\right)^{2}} .
$$

Then the decay rate of the inflaton can be written as

$$
\Gamma_{\phi}=B_{2} \frac{M^{2}}{8 \pi m_{\phi}^{\text {eff }}},
$$

where $B_{2} \approx 2.693$ is a numerical constant.

\section{A.2.3 Sextic potential: $V(\phi)=\frac{V_{0}}{6 M_{\mathrm{pl}}^{6}} \phi^{6}$}

In this case, we numerically found

$$
\beta \approx 1.010, \quad \frac{\left\langle\phi^{2}\right\rangle}{\rho_{\phi}} \approx \frac{12.93}{\left(m_{\phi}^{\mathrm{eff}}\right)^{2}} .
$$

Then the decay rate of the inflaton can be written as

$$
\Gamma_{\phi}=B_{3} \frac{M^{2}}{8 \pi m_{\phi}^{\mathrm{eff}}},
$$

where $B_{3} \approx 4.362$ is a numerical constant.

\section{A.3 Interaction with scalar field $\chi: \mathcal{L}_{\text {int }}=-h \phi^{2} \chi^{2}$}

In this case, the interaction part of the Hamiltonian is given by

$$
\hat{V}=h \phi^{2}(t) \int d^{3} x \hat{\chi} \hat{\chi} .
$$

Let us define $\zeta(t)$ as

$$
\phi^{2}(t)-\left\langle\phi^{2}\right\rangle=\sum_{n=-\infty}^{\infty} \zeta_{n} e^{-i n \omega t} .
$$

Note that $\omega$ is the frequency of $\zeta(t)$. Then the transition rate per unit time and unit volume is

$$
\begin{aligned}
\Gamma & =\frac{h^{2}}{4 \pi} \sum_{n=1}^{\infty}\left|\zeta_{n}\right|^{2} \\
& =\frac{h^{2}}{8 \pi}\left(\left\langle\phi^{4}\right\rangle-\left\langle\phi^{2}\right\rangle^{2}\right) .
\end{aligned}
$$


Introducing the dimensionless quantity $\gamma$ as

$$
\gamma \equiv \frac{\sum_{n=1}^{\infty} n\left|\zeta_{n}\right|^{2}}{\sum_{n=1}^{\infty}\left|\zeta_{n}\right|^{2}}
$$

the inflaton decay rate is given by

$$
\Gamma_{\phi}=\frac{h^{2}}{8 \pi} \gamma \omega \frac{\left\langle\phi^{4}\right\rangle-\left\langle\phi^{2}\right\rangle^{2}}{\rho_{\phi}} .
$$

\section{A.3.1 Quadratic potential: $V(\phi)=\frac{m^{2}}{2} \phi^{2}$}

Using Eq. (54), we have

$$
\gamma=1, \quad \frac{\left\langle\phi^{4}\right\rangle-\left\langle\phi^{2}\right\rangle^{2}}{\rho_{\phi}}=\frac{\phi_{0}^{2}}{4 m^{2}}
$$

Hence $\Gamma_{\phi}$ is given by

$$
\Gamma_{\phi}=\frac{h^{2} \phi_{0}^{2}}{32 \pi m}=\frac{h^{2}}{16 \pi m^{3}} \rho_{\phi}
$$

\section{A.3.2 Quartic potential: $V(\phi)=\frac{\lambda}{4} \phi^{4}$}

Using Eq. (57), we find

$$
\gamma \approx 1.007, \quad \frac{\left\langle\phi^{4}\right\rangle-\left\langle\phi^{2}\right\rangle^{2}}{\rho_{\phi}} \approx \frac{0.50}{\lambda} .
$$

Then the decay rate of the inflaton can be written as

$$
\Gamma_{\phi}=C_{2} \frac{h^{2}}{8 \pi\left(m_{\phi}^{\mathrm{eff}}\right)^{3}} \rho_{\phi},
$$

where $C_{2} \approx 8.86$ is a numerical constant.

\section{A.3.3 Sextic potential: $V(\phi)=\frac{V_{0}}{6 M_{\mathrm{pl}}^{6}} \phi^{6}$}

In this case, we numerically found

$$
\gamma \approx 1.019, \quad \frac{\left\langle\phi^{4}\right\rangle-\left\langle\phi^{2}\right\rangle^{2}}{\rho_{\phi}} \approx \frac{0.73}{V_{0} \phi_{0}^{2}} .
$$

Then the decay rate of the inflaton can be written as

$$
\Gamma_{\phi}=C_{3} \frac{h^{2}}{8 \pi\left(m_{\phi}^{\text {eff }}\right)^{3}} \rho_{\phi},
$$

where $C_{3} \approx 37.26$ is a numerical constant. 


\section{B Calculation of $Q(x)$}

In this section, we will provide explicit form of $Q(x)$ for three types of inflaton potential, quadratic $V \propto \phi^{2}$, quartic $V \propto \phi^{4}$ and sextic $V \propto \phi^{6}$, and for three types of interactions between the inflaton and matter particles, Yukawa interactions, three-point interactions $\mathcal{L}_{\text {int }}=-M \phi \chi^{2}$ and four-point interactions $\mathcal{L}_{\text {int }}=-h \phi^{2} \chi^{2}$.

\section{B.1 Quadratic potential}

If the inflaton potential is quadratic, i.e. $V(\phi)=\frac{m^{2}}{2} \phi^{2}$, then we have $m_{\mathrm{eff}}=m$. Hence the decay rate becomes independent of time for Yukawa interactions and three-point interactions $\mathcal{L}_{\text {int }}=-M \phi \chi^{2}$. In this case, it was shown in Ref. [25] that $Q(x)=-\frac{1}{6} \log x$. Meanwhile, if the dominant decay occurs through the four-point interactions, then the inflaton decay rate decreases as $\Gamma_{\phi} \propto \rho_{\phi}$. From the Friedmann equation $H^{2} \propto \rho_{\phi}$, the ratio $\Gamma_{\phi} / H$ decreases in proportional to $a^{-3 / 2}$. Since $\Gamma_{\phi}$ is smaller than $H$ at initial time, that is, at the end of inflation, $\Gamma_{\phi}$ never becomes larger than $H$. Hence the universe is never reheated only by the four-point interactions, which cannot realize the hot big bang cosmology. Thus we do not consider this case.

\section{B.2 Quartic potential}

If the inflaton potential is quartic, $\rho_{\phi}$ decreases in proportional to $a^{-4}$. Hence the universe expands in the same way as the radiation dominated universe. From the definition of $Q(x)$ (see Eq. (7D), we find

$$
Q(x)=0
$$

for all three types of interactions. Note that unlike in the case of the quadratic potential, the universe can be reheated only by the four-point interactions in this case.

\section{B.3 Sextic potential}

If the inflaton potential is sextic, $\rho_{\phi}$ decays in proportional to $e^{-9 N / 2}$. Then the decay rate of the inflaton evolves as $\propto e^{-3 N / 2}, e^{3 N / 2}$ and constant for Yukawa interactions, threepoint interactions $\mathcal{L}_{\text {int }}=-M \phi \chi^{2}$ and four-point interactions $\mathcal{L}_{\text {int }}=-h \phi^{2} \chi^{2}$, respectively. Since the Hubble parameter decays faster than $e^{-2 N}$, the ratio $\Gamma_{\phi} / H$ grows in time for any interactions. Hence the universe eventually becomes the radiation dominated universe.

Since the most of radiation is produced when $H=\Gamma_{\phi}$, which we checked by a numerical calculation, in what follows, we will use the so-called sudden decay approximation, where the inflaton decays instantaneously when the decay rate becomes equal to the Hubble 
parameter. Under this approximation, the background equations can be written as

$$
\begin{aligned}
& \frac{d \rho_{r}}{d N}+4 \rho_{r}=\frac{\Gamma_{\phi}}{H} \rho_{\phi} \delta\left(N-N_{d}\right), \\
& \frac{d \rho_{\phi}}{d N}+\frac{9}{2} \rho_{\phi}=-\frac{\Gamma_{\phi}}{H} \rho_{\phi} \delta\left(N-N_{d}\right), \\
& H^{2}=\frac{1}{3 M_{\mathrm{pl}}^{2}}\left(\rho_{\phi}+\rho_{r}\right) .
\end{aligned}
$$

Integrating Eq. (92) from $N=0$ which corresponds to $t=t_{c}$ to $N=N_{f}>N_{d}$, we find

$$
N\left(t_{f}, t_{c}\right)=\frac{1}{4} \log \frac{\rho_{c}}{\rho_{f}}+\frac{1}{2} \log \frac{\Gamma_{\phi}\left(t_{c}\right)}{H_{c}}+N_{d} .
$$

Comparing this equation with Eq. (7), $Q(x)$ can be written as

$$
Q=\frac{1}{2} \log \frac{\Gamma_{\phi}\left(N_{d}\right)}{H_{c}}+N_{d}
$$

\section{B.3.1 Yukawa interactions}

In this case, the decay rate can be written as $\Gamma_{\phi}=\Gamma_{\phi}\left(t_{c}\right) e^{-3 N / 2}$. Hence from the equation $H=\Gamma_{\phi}$, we find that $N_{d}$ is given by

$$
N_{d}=-\frac{4}{3} \log \frac{\Gamma_{\phi}\left(t_{c}\right)}{H_{c}}
$$

Substituting this into Eq. (96) gives

$$
Q=\frac{1}{6} \log \frac{\Gamma_{\phi}\left(t_{c}\right)}{H_{c}}
$$

\section{B.3.2 Three-point interactions $\mathcal{L}_{\text {int }}=-M \phi \chi^{2}$}

In this case, the decay rate is $\Gamma_{\phi}=\Gamma_{\phi}\left(t_{c}\right) e^{3 N / 2}$. Then the corresponding $Q$ is given by

$$
Q=\frac{1}{30} \log \frac{\Gamma_{\phi}\left(t_{c}\right)}{H_{c}}
$$

\section{B.3.3 Four-point interactions $\mathcal{L}_{\text {int }}=-h \phi^{2} \chi^{2}$}

In this case, the decay rate is $\Gamma_{\phi}=\Gamma_{\phi}\left(t_{c}\right)$. Then the corresponding $Q$ is given by

$$
Q=\frac{1}{18} \log \frac{\Gamma_{\phi}\left(t_{c}\right)}{H_{c}}
$$




\section{References}

[1] K. Enqvist and M. S. Sloth, Nucl. Phys. B 626, 395 (2002) arXiv:hep-ph/0109214;

[2] D. H. Lyth and D. Wands, Phys. Lett. B 524, 5 (2002) arXiv:hep-ph/0110002];

[3] T. Moroi and T. Takahashi, Phys. Lett. B 522, 215 (2001) [Erratum-ibid. B 539, 303 (2002)] arXiv:hep-ph/0110096.

[4] G. Dvali, A. Gruzinov and M. Zaldarriaga, Phys. Rev. D 69, 023505 (2004) [arXiv:astro-ph/0303591].

[5] L. Kofman, arXiv:astro-ph/0303614.

[6] A. P. S. Yadav and B. D. Wandelt, Phys. Rev. Lett. 100, 181301 (2008) arXiv:0712.1148 [astro-ph]].

[7] E. Komatsu et al. [WMAP Collaboration], arXiv:0803.0547 [astro-ph].

[8] C. Hikage, T. Matsubara, P. Coles, M. Liguori, F. K. Hansen and S. Matarrese, arXiv:0802.3677 [astro-ph].

[9] A. Slosar, C. Hirata, U. Seljak, S. Ho and N. Padmanabhan, arXiv:0805.3580 [astro$\mathrm{ph}$.

[10] A. Curto, E. Martinez-Gonzalez, P. Mukherjee, R. B. Barreiro, F. K. Hansen, M. Liguori and S. Matarrese, arXiv:0807.0231 [astro-ph].

[11] K. Dimopoulos and D. H. Lyth, Phys. Rev. D 69, 123509 (2004) arXiv:hep-ph/0209180.

[12] M. Endo, M. Kawasaki and T. Moroi, Phys. Lett. B 569, 73 (2003) arXiv:hep-ph/0304126].

[13] G. Lazarides, R. R. de Austri and R. Trotta, Phys. Rev. D 70, 123527 (2004) arXiv:hep-ph/0409335.

[14] K. Dimopoulos, D. H. Lyth and Y. Rodriguez, JHEP 0502, 055 (2005) arXiv:hep-ph/0411119.

[15] Y. Rodriguez, Mod. Phys. Lett. A 20, 2057 (2005) arXiv:hep-ph/0411120.

[16] D. Langlois and F. Vernizzi, Phys. Rev. D 70, 063522 (2004) arXiv:astro-ph/0403258].

[17] T. Moroi, T. Takahashi and Y. Toyoda, Phys. Rev. D 72, 023502 (2005) arXiv:hep-ph/0501007. 
[18] T. Moroi and T. Takahashi, Phys. Rev. D 72, $023505 \quad$ (2005) arXiv:astro-ph/0505339.

[19] K. Ichikawa, T. Suyama, T. Takahashi and M. Yamaguchi, Phys. Rev. D 78, 023513 (2008) arXiv:0802.4138 [astro-ph]].

[20] A. A. Starobinsky, JETP Lett. 42 (1985) 152 [Pisma Zh. Eksp. Teor. Fiz. 42 (1985) 124].

[21] M. Sasaki and E. D. Stewart, Prog. Theor. Phys. 95, 71 (1996) arXiv:astro-ph/9507001.

[22] M. Sasaki and T. Tanaka, Prog. Theor. Phys. 99, 763 (1998) arXiv:gr-qc/9801017.

[23] D. H. Lyth, K. A. Malik and M. Sasaki, JCAP 0505, 004 (2005) arXiv:astro-ph/0411220.

[24] T. Suyama and S. Yokoyama, Class. Quant. Grav. 24, 1615 (2007) arXiv:astro-ph/0606228.

[25] T. Suyama and M. Yamaguchi, Phys. Rev. D 77, 023505 (2008) arXiv:0709.2545 [astro-ph]].

[26] T. Suyama and F. Takahashi, arXiv:0804.0425 [astro-ph].

[27] J. Dunkley et al. [WMAP Collaboration], arXiv:0803.0586 [astro-ph].

[28] A. D. Linde, Phys. Lett. B 129, 177 (1983).

[29] A. B. Goncharov and A. D. Linde, Phys. Lett. B 139, 27 (1984).

[30] A. S. Goncharov and A. D. Linde, Class. Quant. Grav. 1, L75 (1984).

[31] H. Murayama, H. Suzuki, T. Yanagida and J. Yokoyama, Phys. Rev. D 50, 2356 (1994) arXiv:hep-ph/9311326.

[32] M. Kawasaki, M. Yamaguchi and T. Yanagida, Phys. Rev. Lett. 85, 3572 (2000) arXiv:hep-ph/0004243].

[33] M. Kawasaki, M. Yamaguchi and T. Yanagida, Phys. Rev. D 63, 103514 (2001) arXiv:hep-ph/0011104.

[34] T. Kawano, arXiv:0712.2351 [hep-th].

[35] T. Kawano and M. Yamaguchi, arXiv:0806.4971 [hep-th].

[36] K. Ichikawa, T. Suyama, T. Takahashi and M. Yamaguchi, in preparation. 
[37] K. Kadota and M. Yamaguchi, Phys. Rev. D 76, 103522 (2007) arXiv:0706.2676 [hep-ph]].

[38] K. Kadota, T. Kawano and M. Yamaguchi, Phys. Rev. D 77, 123516 (2008) arXiv:0802.0525 [hep-ph]].

[39] A. D. Linde, arXiv:hep-th/0503203.

[40] Y. Shtanov, J. H. Traschen and R. H. Brandenberger, Phys. Rev. D 51, 5438 (1995) arXiv:hep-ph/9407247.

[41] P. B. Greene, L. Kofman, A. D. Linde and A. A. Starobinsky, Phys. Rev. D 56, 6175 (1997) arXiv:hep-ph/9705347.

[42] S. Weinberg, The Quantum Theory of Fields volume I, Cambridge University Press 